\title{
Meta-análisis de las elasticidades ingreso y precio de la demanda de gasolina: implicaciones de política pública para América Latina
}

\author{
Luis Miguel Galindo, Joséluis Samaniego, José Eduardo \\ Alatorre, Jimy Ferrer Carbonell y Orlando Reyes
}

RESUMEN

El objetivo de este artículo es estimar, mediante un meta-análisis, el valor promedio de las elasticidades ingreso y precio de la demanda de gasolinas y analizar las causas de la variación en las elasticidades reportadas en la literatura. Los resultados muestran que existe un sesgo de publicación, que la volatilidad de las estimaciones de las elasticidades no se debe exclusivamente a errores de muestreo y que existen factores sistemáticos que explican estas diferencias. Las elasticidades ingreso y precio de la demanda de gasolinas son distintas en el corto y largo plazo, por regiones, y son susceptibles de incluirse en la estimación la flota vehicular y el precio de bienes substitutos, los tipos de datos y métodos de estimación utilizados. La presencia de una baja elasticidad precio sugiere que un impuesto a los combustibles, en un entorno de rápido crecimiento económico, será insuficiente para controlar el aumento del consumo.

PALABRAS CLAVE

CLASIFICACIÓN JEL

AUTORES
Gasolina, oferta y demanda, precios, consumo, modelos econométricos, América Latina

C83, Q41, Q48

Luis Miguel Galindo es Jefe de la Unidad Economía del Cambio Climático de la División de Desarrollo Sostenible y Asentamientos Humanos (DDSAH), CEPAL. luismiguel.galindo@cepal.org

Joséluis Samaniego es Director de la División de Desarrollo Sostenible y Asentamientos Humanos (DDSAH), CEPAL. joseluis.samaniego@cepal.org

José Eduardo Alatorre es Oficial de Asuntos Económicos de la División de Desarrollo Sostenible y Asentamientos Humanos (DDSAH), cEPAL. joseeduardo.alatorre@cepal.org

Jimy Ferrer Carbonell es Oficial de Asuntos Económicos de la División de Desarrollo Sostenible y Asentamientos Humanos (DDSAH), CEPAL. jimy.ferrer@cepal.org

Orlando Reyes es Investigador de la División de Desarrollo Sostenible y Asentamientos Humanos (DDSAH), cEPAL.orlando.reyes@cepal.org 


\section{I}

\section{Introducción}

El consumo de gasolinas es un insumo indispensable para el funcionamiento de las economías modernas, sin embargo, ello también se asocia a la generación de un conjunto de externalidades negativas, tales como tráfico vehicular, contaminación atmosférica y cambio climático (Steenhof y otros, 2006; Galindo, 2008; Reyes, 2009). Diversas proyecciones basadas en escenarios inerciales sugieren que el consumo de gasolinas tendrá un incremento sustancial en los próximos años, intensificando estas externalidades negativas (Calthrop y Proost, 1998; Galindo, 2008; Kim y otros, 2011). Esto es sobre todo relevante en América Latina, donde las externalidades negativas derivadas, por ejemplo, del transporte urbano son ya especialmente intensas y es muy probable que se agudicen en un entorno de rápido crecimiento económico (Alves y Bueno, 2003; Galindo, 2008).

En este contexto, resulta esencial identificar los factores que inciden y sus magnitudes de incidencia en la evolución del consumo de gasolinas. La literatura empírica sobre la demanda de combustible es amplia, muy variada e incluye a distintos países, regiones, períodos e incluso diversas metodologías de estimación. Ello se ha reflejado en la presencia de numerosas estimaciones econométricas sobre las sensibilidades de respuesta de dos factores fundamentales en la evolución del consumo

$\square$ Este estudio forma parte de un conjunto de trabajos realizados en el marco del programa "Política Fiscal y Cambio Climático", con financiamiento de la Agencia Alemana de Cooperación Internacional (GIZ). Las opiniones expresadas en este documento son de exclusiva responsabilidad de los autores y pueden no coincidir con las de la CEPAL, ni reflejar la opinión oficial de los gobiernos de los países, de las instituciones o de los donantes mencionados en el estudio. Los autores agradecen los comentarios de Fernando Filgueira, Gabriel Porcile y José Javier Gómez. Se aplica el descargo usual de los errores. de gasolinas: las elasticidades ingreso y precio. Estas elasticidades son fundamentales para identificar y simular, por ejemplo, las consecuencias de diversos escenarios de crecimiento económico o de la aplicación de un impuesto a las gasolinas. En este sentido, identificarlas apropiadamente resulta fundamental para la construcción de una estrategia de desarrollo sostenible, baja en carbono.

El examen sistemático de este conjunto de información disponible en los distintos estudios sobre la demanda de gasolinas es una tarea compleja, pero puede sintetizarse por medio de un meta-análisis que resume, integra e interpreta los resultados de diversos estudios empíricos (Van den Bergh y otros, 2010; Cumming, 2012; Stanley, 2001) y permite obtener un estimador medio ponderado que incorpora el efecto combinado de los valores encontrados en cada estudio, donde las ponderaciones se asignan teniendo en cuenta la precisión (varianza o error estándar) de los resultados de cada trabajo (Sterne, 2009). Este método permite, además, realizar inferencias generales y explorar la heterogeneidad de los resultados entre los diferentes estudios (Borenstein y otros, 2009; Sáez y otros, 2001).

De este modo, el principal objetivo de este artículo es estimar un meta-análisis de las elasticidades ingreso y precio de la demanda de gasolinas y derivar algunas consecuencias de política pública para América Latina. En el artículo se incluyen cuatro secciones. Luego de la Introducción, en la sección II se presenta la discusión sobre la demanda de gasolinas, los factores que la determinan y una explicación del meta-análisis. En la sesión III se exponen las estimaciones de los coeficientes combinados que se obtienen a partir del meta-análisis y los resultados de las meta-regresiones, asimismo se presentan algunas consideraciones de política pública. Finalmente, en la cuarta sección se entregan las conclusiones y los comentarios generales. 


\section{II}

\section{Meta-análisis de la demanda de gasolinas}

El consumo de gasolinas es un insumo esencial en las economías modernas para el transporte de mercancías y la movilidad de la población. La evolución de su demanda puede modelarse como cualquier otra función de demanda (Deaton y Muellbauer, 1980; Varian, 1993) (ecuación (1)). Esto es, la demanda de gasolinas es función de la evolución del gasto (en ocasiones aproximado por el ingreso), de su precio y de los precios de bienes sustitutos y complementarios, y asimismo de otro conjunto de factores, tales como las características socioeconómicas y demográficas, la flota vehicular, la estructura urbana o regulaciones específicas sobre movilidad urbana y uso del automóvil (Kayser, 2000; Dahl, 2012; Galindo, 2005; Reyes, 2009; Alves y Bueno, 2003):

$$
\operatorname{gas}_{i t}=F\left(y_{i t}, p g_{i t}, p_{i t}, \operatorname{contj}_{i t}\right)
$$

donde gas $_{i t}$ representa la demanda de gasolinas, $y_{i t}$ es la variable de gasto o ingreso, $p g_{i t}$ es su precio relativo, $p_{i t}$ son los precios de los bienes sustitutos y complementarios de las gasolinas, cont $_{j t}$ son otras variables de control que resultan relevantes. El subíndice $t$ representa el tiempo, $i$ representa los diferentes agentes y el subíndice $j$, las variables de control. La literatura económica sugiere que la elasticidad ingreso debe ser positiva y la elasticidad precio, negativa. Esto es, un incremento del gasto total o del ingreso estará acompañado de un crecimiento del consumo de gasolinas, mientras que un aumento de su precio se traduce en una disminución de su demanda. Existe, sin embargo, una gran diversidad de estimaciones econométricas de la ecuación (1) que utilizan distintas especificaciones, métodos, períodos, países, regiones o agentes económicos (Espey, 1998). Ello se refleja en la presencia de distintos valores puntuales en las estimaciones de las elasticidades ingreso y precio de la demanda de gasolinas, lo que induce un importante nivel de incertidumbre desde la óptica de la política pública.

El análisis y la síntesis de estas distintas elasticidades ingreso y precio de la demanda de gasolinas, su volatilidad y posible sesgo de publicación ${ }^{1}$ pueden realizarse sobre

\footnotetext{
${ }^{1}$ El sesgo de publicación existe cuando los estudios no incluidos en el meta-análisis son sistemáticamente diferentes de los que sí lo fueron. Existe evidencia de que esto puede ocurrir, dado que los estudios en que se reportan efectos más altos (por ejemplo, elasticidades) tienen mayor probabilidad de ser publicados que aquellos trabajos donde se encuentran efectos más bajos.
}

la base de un meta-análisis ${ }^{2}$, que es el análisis estadístico del conjunto de investigaciones empíricas y resultados sobre un tema específico, donde se incorpora toda la información disponible para identificar características comunes, efectos medios y las fuentes de heterogeneidad de estos efectos (Stanley, 2001; Lipsey y Wilson, 2001). En este sentido, en cada estudio se tiene estimado un efecto $^{3}\left(\theta_{i}\right)$-magnitud del efecto (size effect) o efecto de tratamiento (treatment effect) — y una varianza $\left(V_{\theta i}\right)$ correspondiente. Sobre la base de estos estadísticos, es posible obtener una media ponderada - conocida como "efecto síntesis" (summary size effect) — de los estudios en que normalmente se le asigna más peso a investigaciones de mayor precisión y donde esta precisión se asocia con el error estándar del efecto ${ }^{4}$ (Lipsey y Wilson, 2001). Sin embargo, las estimaciones de las elasticidades ingreso y precio pueden presentar un exceso de volatilidad - que no es solo consecuencia de un error de muestreo-y tener además un sesgo de publicación derivado del hecho de privilegiar estudios de estimaciones econométricas que son consistentes con la teoría económica y con estimaciones previas o con ambas.

El análisis de meta-regresión (MRA) permite identificar los factores sistemáticos que inciden en la volatilidad de las estimaciones y el sesgo de publicación (Van den Bergh y otros, 2010; Cumming, 2012). Existen diversos tipos de estimaciones de meta-regresiones; en este estudio se utiliza una meta-regresión de tipo Heckman basada en el modelo de efectos mixtos que corrige por el sesgo de publicación (Van den Bergh y otros, 2010; Cumming, 2012; Havranek y otros, 2012). Asimismo, como referencia, se reportan las estimaciones de efectos fijos, aleatorios y de Huber y White que hacen posible corregir problemas de heteroscedasticidad y autocorrelación debido a aglomeraciones productivas (clusters) (Williams, 2000; Wooldridge, 2002). Además, las

\footnotetext{
2 Existen también críticas al meta-análisis, donde se destacan las dificultades de combinar diversos resultados y la generación de una variabilidad de resultados artificiales (Lipsey y Wilson, 2001).

${ }^{3}$ Es común que el objetivo del meta-análisis sea el coeficiente conocido como el efecto puntual (size effect), que identifica la magnitud del efecto considerado en el conjunto de los estudios: $g=\left(\mu_{e}-\mu_{c}\right) / \sigma$. Donde $g$ es el efecto puntual, $\mu_{e}$ y $\mu_{c}$ son las medias del grupo experimental y de control, respectivamente, y $\sigma$ es la desviación estándar del grupo de control (Stanley, 2001).

${ }^{4}$ Existen también meta-análisis multivariados (Lipsey y Wilson, 2001).
} 
estimaciones son ponderadas por la inversa de la desviación estándar para reducir problemas de heterocedasticidad (Lipsey y Wilson, 2001). Cada uno de estos métodos de estimación presenta algunas limitaciones, por ejemplo, los modelos de efectos fijos, aleatorios, mixtos y de Huber-White utilizan diversos supuestos bastante restrictivos (Abreu y Florax, 2005). Al mismo tiempo, la significancia estadística de los modelos de efectos fijos y mixtos debe tomarse con precaución y la estimación con Huber-White no emplea de manera eficiente toda la información disponible (Abreu y Florax, 2005).

El efecto puntual (size effect) de las elasticidades ingreso y precio de la demanda de gasolinas se obtiene de un modelo de regresión representado en la ecuación (2) (Stanley y Jarrel, 1989; Paterson y Canam, 2001):

$$
Y=X \beta+\varepsilon
$$

donde $Y$ es un vector (nx1) que contiene las variables independientes (por ejemplo, la demanda de gasolinas), $X$ es un vector (nxm) de las variables explicativas, $\beta$ es el vector $(m \times 1)$ de coeficientes estimados y $\varepsilon$ es un vector $(\mathrm{nx} 1)$ del término de error.

De este modo, el conjunto de elasticidades estimadas se distribuyen aleatoriamente — en ausencia de sesgo de publicación- en torno del valor real de la elasticidad, con independencia del error estándar (ecuación (3)) (Doucouliagos y Stanley, 2009; Stanley, 2008):

$$
b_{i}=\beta_{0}+w_{i}
$$

donde $b_{i}$ representa las elasticidades estimadas, $\beta_{0}$ el valor real de la elasticidad y $w_{i}$ es el término de error.

En el caso de que las elasticidades estimadas presenten un sesgo de publicación, es posible utilizar una meta-regresión con efectos mixtos y el procedimiento en dos etapas de Heckman (Havranek y otros, 2012). En este procedimiento se utilizan las características de una muestra de control a fin de estimar - con un modelo probit o logit - el sesgo potencial, para incluir en una segunda fase la razón de Mills en la ecuación final (Heckman, 1979; Angrist y Pischke, 2009). En la metaregresión no se dispone de un contra-factual, pero puede aprovecharse la heterogeneidad de la desviación estándar para identificar a la ecuación econométrica, sustituyendo con ello a la razón inversa de Mills (Stanley, 2001).

Así, la ecuación (3) puede reformularse ${ }^{5}$ ante la presencia de sesgo de publicación, incluidos tanto el

\footnotetext{
${ }^{5}$ La aproximación utiliza un polinomio de Taylor (Apostol, 1967) de modo que $b_{1}=\beta_{1}+\sum{ }^{k} \alpha_{k} S E_{i}^{k}+v_{i}$ (Stanley y Doucouliagos, 2012).
}

valor real de la elasticidad como dicho sesgo. Ello se representa en la ecuación (4) (Stanley, 2001; Havranek y otros, 2012; Doucouliagos y Stanley, 2009):

$$
b_{i}=\beta_{0}+\beta_{1}^{*} \operatorname{SE}\left(b_{i}\right)+u_{i}, \quad u_{i} / \operatorname{SE}\left(b_{i}\right) \sim N\left(0, \delta^{2}\right)
$$

donde $b_{i}$ es la elasticidad, $S E$ es el error estándar de cada estimación incluida en el meta-análisis, $\beta_{0}$ representa la media real de la elasticidad, $\beta_{1}$ estima la magnitud del sesgo y $u_{i}$ es el término de error. Un valor significativo de $\beta_{1}$ implica que existe sesgo en las estimaciones (Havranek y otros, 2012; Stanley, 2008). La ecuación (4) se estima por mínimos cuadrados ponderados para reducir posibles problemas de heterocedasticidad (Havranek y otros, 2012; Stanley 2008). Ello se representa en la ecuación (5), donde se identifica al sesgo de publicación en el coeficiente $\beta_{1}$. Esta ecuación permite además observar la significancia estadística de la elasticidad real (coeficiente $\beta_{0}$ ), una vez eliminado el sesgo de publicación. Vale la pena señalar que simulaciones Monte Carlo han mostrado que su valor tiene un sesgo a la baja (Stanley, 2008; Stanley y Doucouliagos, 2011).

$$
\frac{b_{i}}{S E\left(b_{i}\right)}=t_{i}=\frac{\beta_{0} * 1}{S E\left(b_{i}\right)}+\beta_{1}+\xi_{i}, \quad \xi_{i} / S E\left(b_{i}\right) \sim N\left(0, \sigma^{2}\right)(5)
$$

La ecuación (5) puede modificarse para incorporar la presencia de volatilidad de las estimaciones entre estudios (Havranek y otros, 2012). De esta manera, la ecuación (6) permite obtener la dirección, magnitud y significancia estadística del sesgo mediante el coeficiente $\beta_{1}$ y la significancia estadística, más allá del sesgo de publicación y del efecto medio estimado por medio del coeficiente $\beta_{0}$ (Havranek y otros, 2012):

$t_{i j}=\frac{\beta_{0} * 1}{S E\left(b_{i j}\right)}+\beta_{1}+\xi_{j}+\varepsilon_{i j}, \quad \varepsilon_{i j} / S E\left(b_{i j}\right), \xi_{j} \sim N(0, \theta)$

donde $i$ y $j$ son subíndices que representan las estimaciones y los estudios. Los errores totales $\left(\zeta_{i j}\right)$ incluyen entonces la parte correspondiente a los efectos aleatorios al nivel de los estudios $\left(\xi_{j}\right)$ y los errores al nivel de estimación $\left(\varepsilon_{i j}\right)$. Donde $\operatorname{var}\left(\zeta_{i j}\right)=\psi+\theta$ y $\psi$ representa la varianza entre estudios (between studies variance) y $\theta$ es la varianza dentro de los estudios (within studies). La varianza de estos dos errores se suma debido a que se consideran independientes. 
Por lo tanto, la magnitud real de la elasticidad media $\left(\beta_{0}\right)$ se puede estimar con una ecuación aumentada, conocida como la meta-regresión de Heckman por efectos mixtos, que contiene una parte de efectos fijos capturada en $\beta_{1}$ y una parte aleatoria $\left(\zeta_{i}\right)^{6}$ (Stanley y Doucouliagos, 2012) y donde se asume que la relación entre los errores estándar y el sesgo de publicación es cuadrática (Stanley, 2008; Stanley y Doucouliagos, 2011):

$$
\begin{gathered}
t_{i j}=\frac{\beta_{0} * 1}{S E\left(b_{i j}\right)}+\beta_{1} S E+\xi_{j}+\varepsilon_{i j}, \quad \xi_{j} / \operatorname{SE}\left(b_{i j}\right) \sim N(0, \psi), \\
\varepsilon_{i j} / \operatorname{SE}\left(b_{i j}\right), \xi_{j} \sim N(0, \theta)
\end{gathered}
$$

donde $\beta_{0}$ mide la magnitud de la elasticidad promedio corregida por el sesgo y $\beta_{1}$ representa la magnitud del sesgo. Es posible, además, identificar algunos de los factores que inciden en la volatilidad de las estimaciones y su sesgo de publicación mediante la ecuación (8) (Havranek y otros, 2012; Van den Bergh y otros, 2010; Phillips y Goss, 1995):

${ }^{6}$ Es similar a un modelo aleatorio de datos panel (Havranek y otros, 2012).

$$
t_{i j}=\frac{\beta_{0} * 1}{S E\left(e_{i j}\right)}+\beta_{1}+\sum_{k=1}^{k} \alpha_{k} Z_{i k}+\xi_{j}+\varepsilon_{i j}
$$

donde $\beta_{1}$ representa el sesgo de publicación, $\alpha_{k}$ son los coeficientes del vector $Z_{i k}$ de la meta-regresión, que incluye las variables que inciden en la volatilidad y no están correlacionadas con los procesos de selección de los estudios (Doucouliagos y Stanley, 2009; Stanley y Jarrel, 1989). El subíndice $i$ indica el valor que toma la variable en cada estudio $i$. En el vector $Z_{i k}$ se incluyen varios factores, como por ejemplo, que las estimaciones correspondan a un país de la Organización para la Cooperación y el Desarrollo Económicos (OCDE) (excluidos Chile y México), la flota vehicular, los precios de los bienes sustitutos o complementarios como variable explicativa en la estimación de la demanda de gasolinas, y el método de estimación utilizado, diferenciando entre datos panel, de sección cruzada y de series de tiempo.

La prueba de la significancia estadística de $\alpha_{0}$ se conoce en la literatura como la prueba de la gráfica de embudo de asimetría (funnel graph's asymetry test) (FAT) y la significancia estadística de $\beta_{0}$ se conoce como la prueba de la precisión de efectos (precision effect test) (PET) (Doucouliagos y Stanley, 2009; Stanley, 2005 y 2008). De este modo, el análisis de la meta-regresión (ecuación (8)) se conoce como el análisis FAT-PET-MRA (Doucouliagos y Stanley, 2009; Stanley, 2008).

\section{III}

\section{Las meta-regresiones y las políticas públicas}

La base de datos empleada consiste en 63 estudios de la literatura internacional sobre la demanda de gasolina, donde se obtuvieron 227 estimaciones de la elasticidad ingreso y 343 de la elasticidad precio. Los estudios utilizados abarcan el período 1960-2013 y provienen de la base de datos EconLit, Jstor y ProQuest, entre otros. Los trabajos seleccionados están publicados en español e inglés ${ }^{7}$.

Las elasticidades ingreso y precio de la demanda de gasolinas se dividieron entre estimaciones de corto y de largo plazo. Es común esperar que la sensibilidad sea mayor en el largo plazo, ya que los consumidores

\footnotetext{
${ }^{7}$ Los autores ponen a disposición la lista de los artículos usados en el meta-análisis.
}

pueden ajustar estructuralmente, por ejemplo, a la nueva estructura de precios relativos. En el cuadro 1 se presenta un resumen de las estadísticas de las elasticidades ingreso y precio de la demanda de gasolinas reportadas en la literatura internacional.

El conjunto de las elasticidades estimadas en los estudios muestra en general una alta volatilidad. Esto es, las estimaciones econométricas — por efectos aleatorios- de la elasticidad ingreso de corto y de largo plazo tienen una media ponderada por la desviación entandar de 0,30 y de 0,62, respectivamente (véase el cuadro 2). Asimismo, las estimaciones - por efectos aleatorios- de la elasticidad precio de corto y de largo plazo tienen una media ponderada por la desviación estándar de -0,20 y de -0,39, respectivamente (véase el cuadro 3). Estas estimaciones de las elasticidades precio 
CUADRO 1

Estadísticas de las elasticidades de la demanda de gasolinas en la literatura internacional

\begin{tabular}{|c|c|c|c|c|c|}
\hline Variable & $\mathrm{N}^{\mathrm{o}}$ de observaciones & Promedio & Desviación estándar & Mínimo & Máximo \\
\hline \multicolumn{6}{|l|}{ Elasticidad ingreso } \\
\hline Elasticidad de largo plazo & 119 & 0,63 & 0,34 & 0,04 & 1,19 \\
\hline Elasticidad de corto plazo & 108 & 0,34 & 0,19 & 0,01 & 0,94 \\
\hline \multicolumn{6}{|l|}{ Elasticidad precio } \\
\hline Elasticidad de largo plazo & 213 & $-0,44$ & 0,28 & $-1,63$ & $-0,32$ \\
\hline Elasticidad de corto plazo & 130 & $-0,21$ & 0,19 & $-1,03$ & 0,31 \\
\hline
\end{tabular}

Fuente: Elaboración propia.

CUADRO 2

Resultado del meta-análisis de la elasticidad ingreso de la demanda de gasolinas

\begin{tabular}{lccccc}
\hline \multirow{2}{*}{ Parámetro } & \multicolumn{2}{c}{ Efectos fijos } & & \multicolumn{2}{c}{ Efectos aleatorios } \\
\cline { 2 - 3 } \cline { 5 - 6 } & Largo plazo & Corto plazo & & Largo plazo & Corto plazo \\
\hline$\theta$ & 0,551 & 0,268 & 0,625 & 0,303 \\
$\sigma_{\theta}$ & 0,002 & 0,001 & 0,031 & 0,017 \\
$\theta+1,96^{*} \sigma_{\theta}$ & 0,554 & 0,270 & & 0,336 \\
$\theta-1,96^{*} \sigma_{\theta}$ & 0,548 & 0,266 & & 0,565 & 0,269 \\
$Z_{\theta}=\theta / \sigma_{\theta}$ & 324,52 & 3,691 & & 0,41 & 17,77 \\
$P=2[1-(\Phi(|Z|))]$ & 0,000 & 0,000 & & 0,000 & 0,000 \\
\hline
\end{tabular}

Fuente: Elaboración propia sobre la base de los resultados de las estimaciones del meta-análisis.

CUADRO 3

Resultado del meta-análisis de la elasticidad precio de la demanda de gasolinas

\begin{tabular}{lccrrr}
\hline \multirow{2}{*}{ Parámetro } & \multicolumn{2}{c}{ Efectos fijos } & & \multicolumn{2}{c}{ Efectos aleatorios } \\
\cline { 2 - 3 } \cline { 5 - 6 } & Largo plazo & Corto plazo & & Largo plazo & Corto plazo \\
\hline$\theta$ & $-0,131$ & $-0,108$ & & $-0,397$ & $-0,204$ \\
$\sigma_{\theta}$ & 0,003 & 0,002 & & 0,015 & 0,021 \\
$\theta+1,96^{*} \sigma_{\theta}$ & $-0,136$ & $-0,112$ & & $-0,427$ & $-0,245$ \\
$\theta-1,96^{*} \sigma_{\theta}$ & $-0,125$ & $-0,105$ & & $-0,367$ & $-0,163$ \\
$Z_{\theta}=\theta / \sigma_{\theta}$ & 44,77 & 63,95 & & 26,05 & 9,75 \\
$P=2[1-(\Phi(|Z|))]$ & 0,000 & 0,000 & & 0,000 & 0,000 \\
\hline
\end{tabular}

Fuente: Elaboración propia sobre la base de los resultados de las estimaciones del meta-análisis.

están en el rango de las elasticidades precio promedio reportadas en los meta-análisis de Espey (1998); Hanly y otros (2002); Brons y otros (2008).

Los histogramas y los gráficos de densidad de Kernel de las elasticidades ingreso y precio muestran que las distribuciones no son simétricas. En el caso de la elasticidad ingreso es más notorio el sesgo positivo en las estimaciones de corto plazo (véase el gráfico 1), mientras que en la elasticidad precio el sesgo es más evidente en las estimaciones de largo plazo (véase el gráfico 2).

Asimismo, las gráficas de embudo de asimetría —donde las elasticidades ingreso y precio se reportan en el eje horizontal y el nivel de precisión de las estimaciones 
GRÁFICO 1

Distribución de las estimaciones de elasticidad ingreso de la demanda de gasolinas

A. Elasticidad ingreso de largo plazo

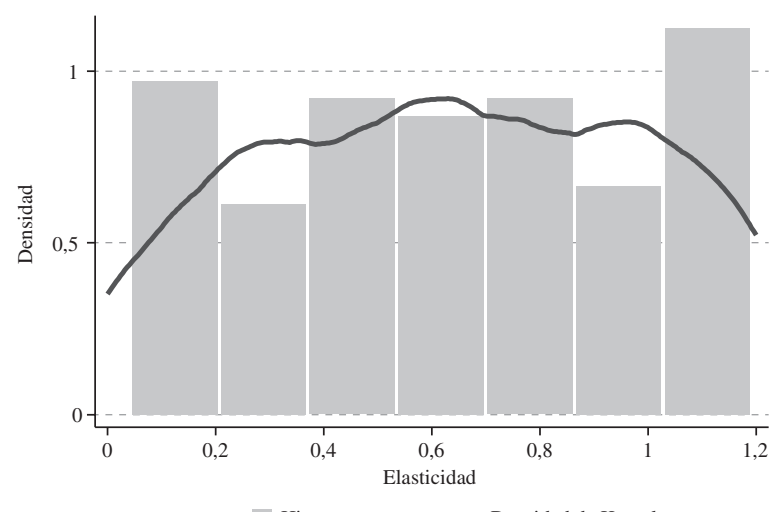

Histograma Densidad de Kernel
B. Elasticidad ingreso de corto plazo

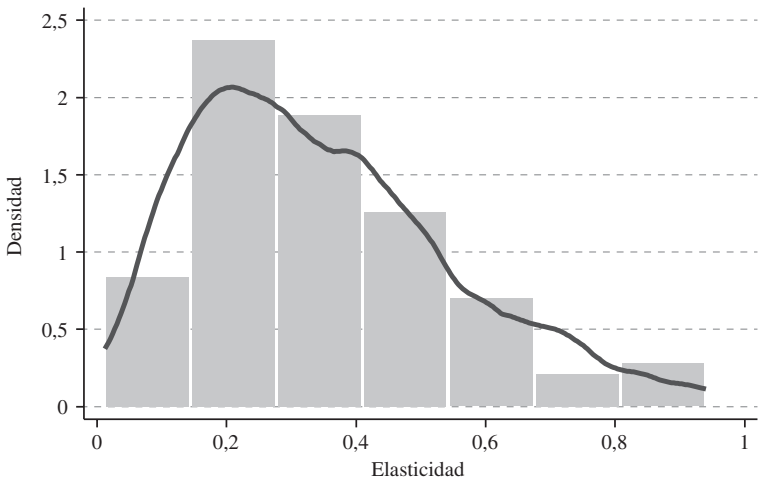

Histograma Densidad de Kernel

Fuente: Elaboración propia.

A. Elasticidad precio de largo plazo

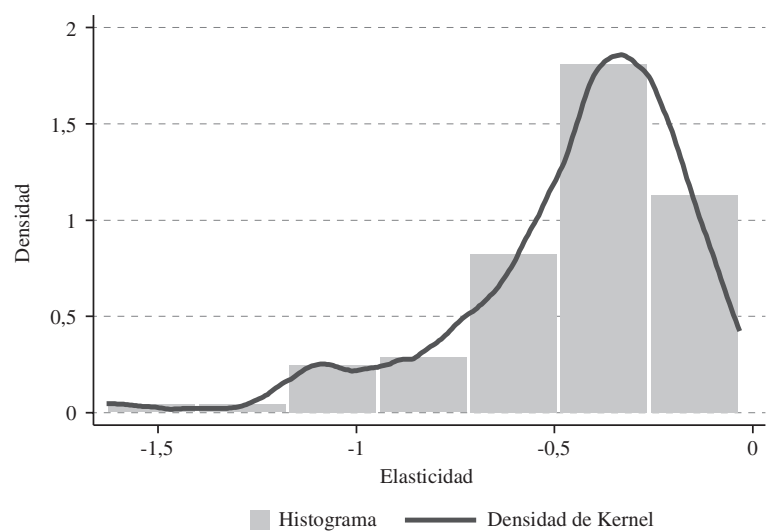

Fuente: Elaboración propia.

en el eje vertical ${ }^{8}$ (Abreu y Florax, 2005; Sterne y otros, 2000)— muestran que puede existir un sesgo de publicación importante ${ }^{9}$ (véanse los gráficos 3 y 4 ). En

${ }^{8}$ La forma de las gráficas de embudo de asimetría, en ausencia de sesgo, depende de la variable escogida para los ejes; en algunos casos se suele utilizar el error estándar, el inverso del error estándar, la varianza, el inverso de la varianza, el tamaño de la muestra, el logaritmo del tamaño de la muestra para el eje vertical, y la magnitud del efecto en el eje horizontal, siendo probablemente el error estándar la mejor opción para el eje vertical (Sterne y Egger, 2001).

${ }^{9}$ El sesgo de publicación ha sido relacionado frecuentemente con la asimetría en las gráficas de embudo; sin embargo, en la literatura se
B. Elasticidad precio de corto plazo

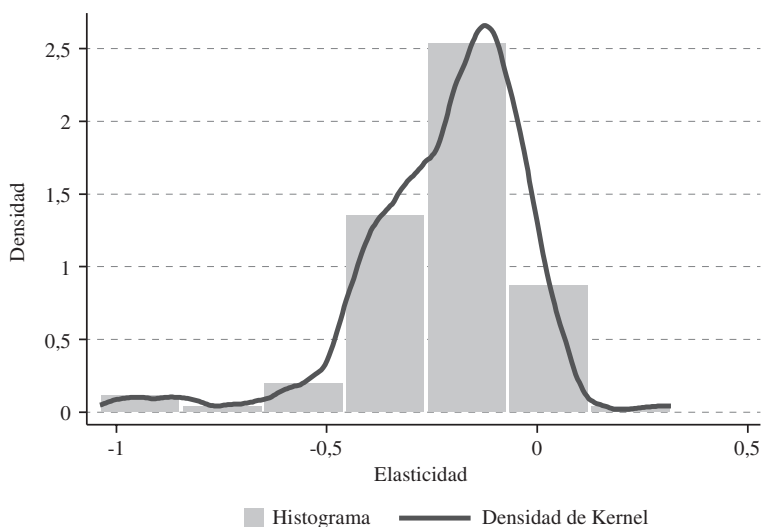

particular, se observa que la distribución de los puntos que representan la relación entre las elasticidades y sus respectivas desviaciones estándar no es simétrica dentro de los límites de confianza del 95\%. Ello sugiere que podría existir una tendencia estadísticamente significativa a publicar estudios que estiman elasticidades ingreso y precio con valores altos.

reporta que existen otras fuentes de la asimetría como los sesgos de selección, la heterogeneidad entre los tamaños de las muestras y la irregularidad de los datos, entre otros factores (Egger y otros, 1997). 


\section{Gráficas de embudo de las elasticidades ingreso y precio de la demanda de gasolinas}

A. Elasticidades ingreso de largo plazo

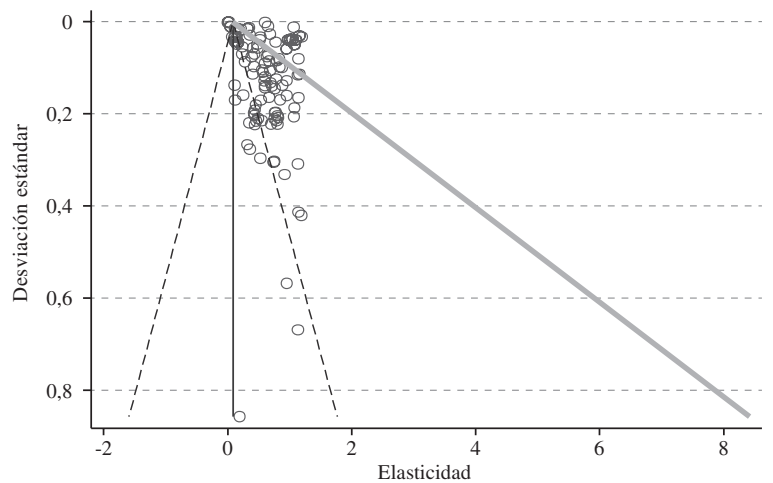

B. Elasticidades ingreso de corto plazo

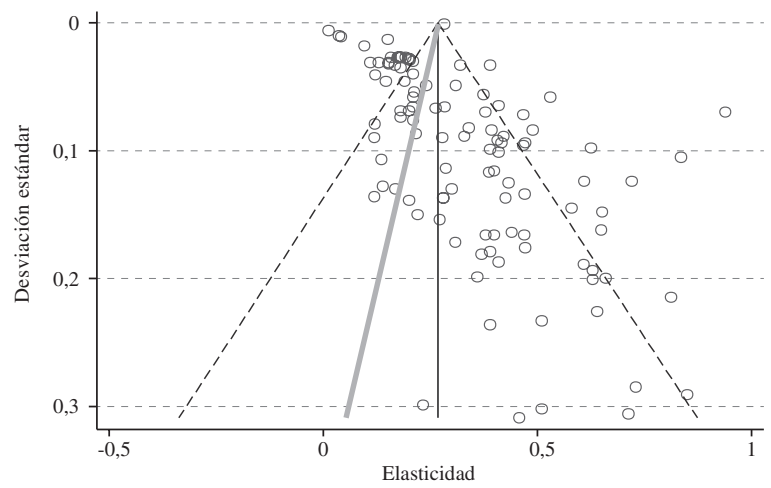

Fuente: Elaboración propia.

Nota: Las líneas punteadas que aparecen en los gráficos de embudo indican los límites de confianza del 95\% y la línea gris el ajuste de la regresión que relaciona las elasticidades precio e ingreso de las gasolinas con sus respectivas desviaciones estándar.

\section{Gráficas de embudo de las elasticidades ingreso y precio de la demanda de gasolinas}

A. Elasticidades precio de largo plazo

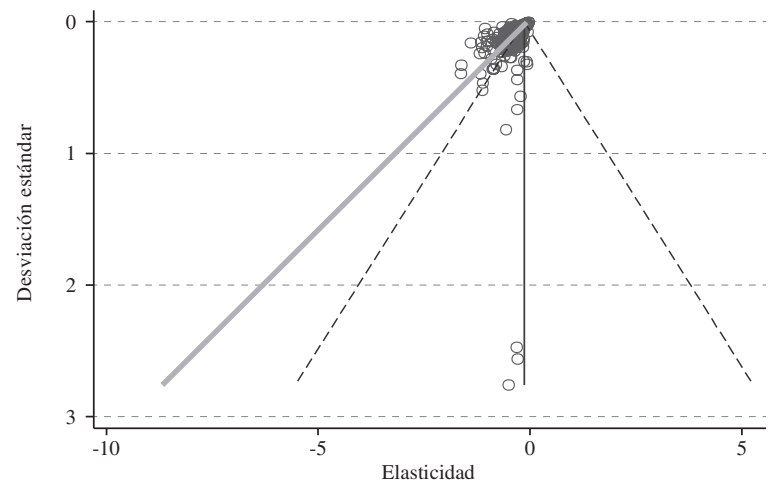

B. Elasticidades precio de corto plazo

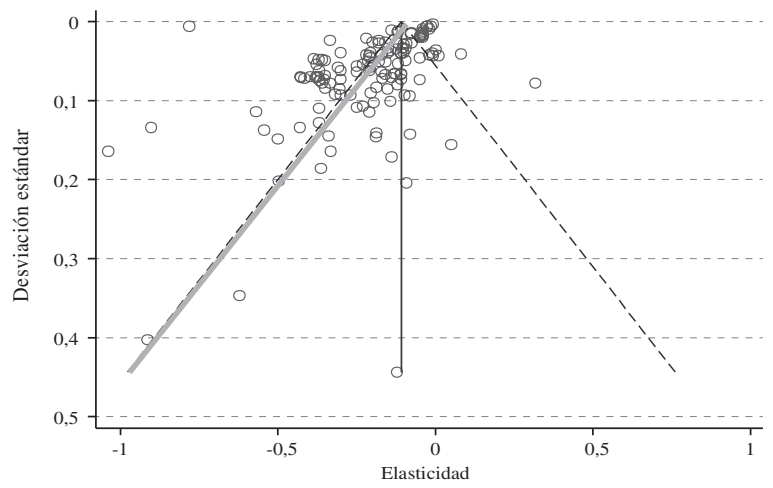

Fuente: Elaboración propia.

Nota: Las líneas punteadas que aparecen en los gráficos de embudo indican los límites de confianza del 95\% y la línea gris el ajuste de la regresión que relaciona las elasticidades precio e ingreso de la gasolina con sus respectivas desviaciones estándar.

El conjunto de la evidencia disponible sugiere entonces la posibilidad de que la volatilidad entre efectos estimados de los estudios no se debe exclusivamente a errores de muestreo y que existe, por lo tanto, una volatilidad originada en factores sistemáticos (Van den Bergh y otros, 2010). El estadístico $Q$ y su valor $p$ rechazan la hipótesis nula de que la distribución es homogénea para las estimaciones de la elasticidad ingreso y precio de corto y largo plazo (véanse los cuadros 4 y 5). El valor $p$ del estadístico $Q$ es menor que el $1 \%$ del nivel de significancia para las pruebas de las estimaciones de elasticidad ingreso (véase el cuadro 4) y precio (véase el cuadro 5) tanto de largo como de corto plazo.

El estadístico $I^{2}$ indica que más del $90 \%$ de la variación observada en la magnitud de los efectos es atribuible a la heterogeneidad entre los estudios, lo que 
Pruebas de heterogeneidad de la elasticidad ingreso de la demanda de gasolinas

\begin{tabular}{lrr}
\hline Prueba de heterogeneidad & Largo plazo & Corto plazo \\
\hline$Q$ & 15610,00 & 3691,00 \\
valor $p$ & 0,000 & 0,000 \\
$\tau^{2}$ & 0,093 & 0,021 \\
$\tau$ & 0,306 & 0,146 \\
$I^{2}$ & $99,2 \%$ & $97,1 \%$
\end{tabular}

Fuente: Elaboración propia sobre la base de los resultados de las estimaciones del meta-análisis.

Nota: $\tau^{2}$ se define como la varianza de la magnitud de los efectos e indica la varianza entre los estudios. $I^{2}$ indica la proporción de la variación observada en la magnitud de los efectos atribuible a la heterogeneidad entre los estudios.

CUADRO 5

Pruebas de heterogeneidad de la elasticidad precio de la demanda de gasolinas

\begin{tabular}{lrr}
\hline Prueba de heterogeneidad & Largo plazo & Corto plazo \\
\hline$Q$ & 3341,34 & 15465,27 \\
valor $p$ & 0,000 & 0,000 \\
$\tau^{2}$ & 0,031 & 0,051 \\
$\tau$ & 0,175 & 0,225 \\
$I^{2}$ & $93,7 \%$ & $99,2 \%$
\end{tabular}

Fuente: Elaboración propia sobre la base de los resultados de las estimaciones del meta-análisis.

Nota: $\tau^{2}$ se define como la varianza de la magnitud de los efectos e indica la varianza entre los estudios. $I^{2}$ indica la proporción de la variación observada en la magnitud de los efectos atribuible a la heterogeneidad entre los estudios.

denota que la variación de las elasticidades ingreso y precio de la demanda de gasolinas se debe casi en su totalidad a la diferencia de los estudios incluidos en el meta-análisis. Ello sugiere aplicar entonces técnicas de meta-regresión para identificar las razones de la variabilidad de las elasticidades (véase el cuadro 8).

Las estimaciones de la ecuación (6) muestran, sobre la base de la prueba de razón de máxima verosimilitud, que se rechaza la hipótesis nula de homogeneidad de las estimaciones (véase el cuadro 6). Esto sugiere que existe una importante heterogeneidad en las elasticidades ingreso y precio y que, por lo tanto, resulta adecuado el modelo de efectos mixtos. Los resultados muestran, para la elasticidad ingreso de largo plazo, que existe un sesgo positivo, mientras que para la elasticidad de corto plazo el sesgo es negativo, si bien este sesgo de corto plazo no es estadísticamente significativo. Los resultados para la elasticidad precio de corto y largo plazo (véase el cuadro 6) indican que existe un sesgo negativo estadísticamente significativo; ello es consistente con lo que se reporta en las gráficas de embudo de asimetría. Asimismo, los resultados de las estimaciones de la ecuación (6) denotan que el efecto o la elasticidad real es estadísticamente distinta de cero en todos los casos (el coeficiente 1/SE es estadísticamente significativo al $1 \%$ ). Esto es, las elasticidades ingreso y precio son factores que inciden en la trayectoria del consumo de gasolinas en el corto y el largo plazo.

Las estimaciones de la meta-regresión de Heckman (ecuación (7)) muestran que las elasticidades ingreso de corto y largo plazo son de 0,26 y 0,46 , respectivamente, una vez corregido el sesgo de publicación. A su vez, las elasticidades precio de corto y largo plazo son de $-0,10$ y $-0,31$, respectivamente, incorporando la corrección del sesgo (véase el cuadro 7). Las pruebas de razón de verosimilitud rechazan la hipótesis nula de que la volatilidad es solo consecuencia del error de muestreo y, por tanto, es adecuado el uso del modelo de efectos mixtos.

En la literatura internacional sobre la demanda de gasolinas se sugiere que existen diversos factores que inciden en la volatilidad de las estimaciones de las elasticidades ingreso y precio de tal demanda, además de la diferencia ya considerada entre elasticidades de corto y largo plazo. En principio, pueden considerarse los siguientes factores: 
CUADRO 6

Prueba de sesgo de publicación

\begin{tabular}{|c|c|c|c|c|}
\hline \multirow{2}{*}{ Variable dependiente: $t$ estadístico } & \multicolumn{2}{|c|}{ Elasticidad ingreso } & \multicolumn{2}{|c|}{ Elasticidad precio } \\
\hline & Largo plazo & Corto plazo & Largo plazo & Corto plazo \\
\hline Constante & $\begin{array}{c}0,767 \\
(1,039)\end{array}$ & $\begin{array}{l}-0,697 \\
(0,579)\end{array}$ & $\begin{array}{l}-1,500 * * * \\
(0,268)\end{array}$ & $\begin{array}{l}-1,973^{*} \\
(1,171)\end{array}$ \\
\hline$(1 / S E)$ & $\begin{array}{l}0,456^{* * *} \\
(0,019)\end{array}$ & $\begin{array}{l}0,270^{* * * *} \\
(0,005)\end{array}$ & $\begin{array}{l}-0,234 * * * \\
(0,020)\end{array}$ & $\begin{array}{l}-0,086^{* * *} * \\
(0,022)\end{array}$ \\
\hline $\mathrm{N}^{\mathrm{o}}$ de observaciones & 119 & 108 & 213 & 130 \\
\hline Prueba de razón de verosimilitud & $561,15 * * *$ & $2185,27 * * *$ & $130,07 * * *$ & $14,47 * * *$ \\
\hline
\end{tabular}

Fuente: Elaboración propia a partir de las estimaciones del modelo de efectos mixtos.

Nota: Los valores entre paréntesis son los errores estándar. Los asteriscos $* * *, * *$ y $*$ indican significancia al $1 \%, 5 \%$ y $10 \%$, respectivamente. La prueba de significancia conjunta se hace mediante la $\chi^{2}$. SE corresponde al error estándar.

CUADRO 7

Elasticidad ingreso y precio de la demanda de gasolinas corregida por sesgo

\begin{tabular}{|c|c|c|c|c|}
\hline \multirow{2}{*}{ Variable dependiente: estadístico $t$} & \multicolumn{2}{|c|}{ Elasticidad ingreso } & \multicolumn{2}{|c|}{ Elasticidad precio } \\
\hline & Largo plazo & Corto plazo & Largo plazo & Corto plazo \\
\hline Elasticidad corregida $(1 / S E)$ & $\begin{array}{l}0,461 * * * \\
(0,017)\end{array}$ & $\begin{array}{l}0,267 * * * \\
(0,006)\end{array}$ & $\begin{array}{l}-0,314 * * * \\
(0,014)\end{array}$ & $\begin{array}{l}-0,104 * * * \\
(0,018)\end{array}$ \\
\hline$S E$ & $\begin{array}{c}4,493 \\
(5,178)\end{array}$ & $\begin{array}{c}4,646 \\
(4,315)\end{array}$ & $\begin{array}{l}-1,004 * * \\
(0,520)\end{array}$ & $\begin{array}{r}-10,978 \\
(9,828)\end{array}$ \\
\hline $\mathrm{N}^{\mathrm{o}}$ de observaciones & 119 & 108 & 213 & 130 \\
\hline Prueba de razón de verosimilitud $\left(\chi^{2}\right)$ & $711,50 * * *$ & $2323,50 * * *$ & $497,31 * * *$ & $36,06^{* * * *}$ \\
\hline
\end{tabular}

Fuente: Elaboración propia sobre la base de las estimaciones del modelo de efectos mixtos.

Nota: Los valores entre paréntesis son los errores estándar. $* * *, * *$ y $*$ indican rechazo al $1 \%, 5 \%$ y $10 \%$, respectivamente de nivel de significancia. La prueba de significancia conjunta se hace a través de la $\chi^{2}$. $S E$ corresponde al error estándar.

i) Las magnitudes de las elasticidades ingreso y precio de la demanda de gasolinas son distintas por países o regiones (Pock, 2007; Bentzen, 1994; Sterner, Dahl y Franzén, 1992). Por lo cual, se espera una mayor elasticidad ingreso de la demanda de gasolinas en los países de renta media que en los países desarrollados y una elasticidad precio de la demanda de gasolinas menos elástica en los países en desarrollo con respecto a los países desarrollados (Brons y otros, 2008; Havranek y otros, 2012). Esta diferencia en las elasticidades precio de la demanda de gasolinas puede explicarse, entre otros factores, por la mayor disponibilidad de sustitutos del transporte privado en países desarrollados.

ii) Las magnitudes de las elasticidades ingreso y precio se modifican de acuerdo con las características y la evolución de la flota vehicular (Espey, 1998; Brons y otros, 2008). Es decir, la demanda de gasolinas puede derivarse de las características de la flota vehicular (por ejemplo, el gasto en combustible por kilómetro) y de los kilómetros recorridos (Brons y otros, 2008):

$$
G A S_{i t}=\sum_{i=1}^{i=n} K M_{i t} C A R_{i t}
$$

donde $G A S_{i t}$ representa el consumo total de gasolinas, $K M_{i t}$ son los kilómetros recorridos por la flota vehicular, $C A R_{i t}$ es dicha flota, $n$ es el número total de vehículos y los subíndices $i$ y $t$ representan los vehículos y el tiempo, respectivamente. En la literatura económica se establece también que la demanda de automóviles es una función, entre otros factores, de la evolución del gasto o del ingreso y los precios relativos de los vehículos. De esta manera, la inclusión de la flota vehicular en la ecuación de demanda de gasolinas induce que el efecto del ingreso en dicha demanda tenga un canal adicional representado por el parque vehicular. Así, la inclusión de la tenencia de automóviles y de las características de la flota vehicular (eficiencia) deriva 
en estimaciones más inelásticas de la demanda con respecto al ingreso en el corto plazo y, posiblemente, en el largo plazo (Espey, 1998).

iii) Las magnitudes de las elasticidades precio de la demanda de gasolinas se modifican al incluirse los precios de otros combustibles sustitutos en la especificación de dicha demanda, como el etanol o biodiese ${ }^{10}$ (Dahl, 1992), o al incluirse el precio del transporte público. Por ejemplo, Goodwin (1992) hace ver que el aumento del precio del transporte público reduce su uso y, por tanto, existen canales de transmisión con el nivel de uso del transporte privado.

iv) Las magnitudes de las elasticidades ingreso y precio son diferentes, dependiendo del tipo de datos y los métodos de estimación aplicados (Graham y Glaister, 2002; Espey, 1998). Vale decir, que resulta común que las estimaciones de datos de panel o sección cruzada se traduzcan en coeficientes menores que aquellos coeficientes estimados con series de tiempo. En los cuadros 9 y 10 se reportan los resultados de la meta-regresión mediante diferentes métodos de estimación, donde se identifican los factores que determinan la heterogeneidad en la magnitud de las

${ }^{10}$ Se consideró incluir el gas natural en el análisis de la demanda de gasolinas, debido a su importancia como sustituto para el transporte en algunos países de América Latina; sin embargo, no se contó con un número suficiente de estudios para incorporarlos en la meta-regresión. elasticidades ingreso y precio de la demanda de gasolinas de largo y corto plazo.

Las estimaciones para las elasticidades ingreso y precio por medio de los métodos de efectos fijos, aleatorios y mixtos tipo Heckman se presentan en el cuadro 11. La evidencia disponible sugiere, en general, que el modelo de efectos mixtos ${ }^{11}$ es el más apropiado.

De este modo, los resultados obtenidos permiten realizar las siguientes inferencias:

- Las magnitudes de las elasticidades ingreso y precio de corto y largo plazo de la demanda de gasolinas son distintas por países o regiones (Pock, 2007; Bentzen, 1994; Sterner, Dahl y Franzén, 1992). La elasticidad ingreso de largo plazo para países de la oCDE, excluidos Chile y México, son menores $(0,55)$ que aquellas referidas a América Latina $(0,69)$, mientras que las elasticidades precio de largo plazo son mayores, en valores absolutos, en los países de la oCDE $(-0,41)$, excluidos Chile y México, que aquellas identificadas para América Latina $(-0,31)$ (véase el cuadro 12). Las elasticidades ingreso y precio de corto plazo son en cambio similares entre las diferentes regiones.

\footnotetext{
${ }^{11}$ Por ejemplo, Havranek y otros (2012) muestran que con el modelo de efectos mixtos se obtienen elasticidades precio sustancialmente más bajas que aquellas obtenidas en otros meta-análisis de precio (Brons y otros, 2008; Espey, 1998).
}

CUADRO 8

\section{Descripción de las variables incluidas en la meta-regresión}

\begin{tabular}{|c|c|}
\hline Variable & Descripción \\
\hline estadístico $t$ & Valor del estadístico $t$ para las estimaciones reportadas en cada estudio. \\
\hline $1 / S E$ & $\begin{array}{l}\text { Variable que mide la precisión de la estimación como el inverso de la desviación estándar de la elasticidad } \\
\text { estimada. }\end{array}$ \\
\hline OCDE & $\begin{array}{l}\text { Variable cualitativa que toma el valor de } 1 \text { si el estudio incluido se realizó para un país (ciudad, estado o región) } \\
\text { de la ocDE y } 0 \text { de lo contrario. }\end{array}$ \\
\hline América Latina & $\begin{array}{l}\text { Variable cualitativa que toma el valor de } 1 \text { si el estudio incluido se realizó para un país (ciudad, estado o región) } \\
\text { de América Latina y } 0 \text { de lo contrario. }\end{array}$ \\
\hline Parque vehicular & $\begin{array}{l}\text { Variable ficticia que toma el valor de } 1 \text { si el modelo reportado en el estudio incluye como variable explicativa } \\
\text { el parque vehicular y } 0 \text { de lo contrario. }\end{array}$ \\
\hline Sustituto & $\begin{array}{l}\text { Variable ficticia que toma el valor de } 1 \text { si el modelo reportado en el estudio incluye como variable explicativa } \\
\text { el precio de otro combustible y } 0 \text { de lo contrario. }\end{array}$ \\
\hline Sección cruzada & $\begin{array}{l}\text { Variable ficticia que toma el valor de } 1 \text { si la información del modelo corresponde a sección cruzada y } 0 \text { de lo } \\
\text { contrario. }\end{array}$ \\
\hline Nacional & Variable ficticia que toma el valor de 1 si el estudio incluido es realizado a nivel nacional y 0 de lo contrario. \\
\hline Dinámico & $\begin{array}{l}\text { Variable ficticia que toma el valor de } 1 \text { si la especificación del modelo corresponde a un modelo dinámico y } 0 \\
\text { de lo contrario. }\end{array}$ \\
\hline
\end{tabular}

Fuente: Elaboración propia.

Nota: ocDE: Organización para la Cooperación y el Desarrollo Económicos.

$S E$ corresponde al error estándar. 
CUADRO 9

Meta-regresión: determinantes de la elasticidad ingreso de la demanda de gasolinas

\begin{tabular}{|c|c|c|c|c|c|c|c|c|}
\hline \multirow{2}{*}{ Parámetro } & \multicolumn{2}{|c|}{ Efectos mixtos } & \multicolumn{2}{|c|}{ Hubber-White } & \multicolumn{2}{|c|}{ Efectos aleatorios } & \multicolumn{2}{|c|}{ Efectos fijos } \\
\hline & Largo plazo & Corto plazo & Largo plazo & Corto plazo & Largo plazo & Corto plazo & Largo plazo & Corto plazo \\
\hline $1 / S E$ & $\begin{array}{l}0,473 * * * \\
(0,014)\end{array}$ & $\begin{array}{l}0,271 * * * \\
(0,005)\end{array}$ & & & & & & \\
\hline OCDE & $\begin{array}{l}-9,263 * * * \\
(1,970)\end{array}$ & $\begin{array}{l}-2,436^{*} \\
(1,438)\end{array}$ & $\begin{array}{l}-0,310 * * * \\
(0,093)\end{array}$ & $\begin{array}{l}-0,019 \\
(0,049)\end{array}$ & $\begin{array}{l}-0,341 * * * * \\
(0,065)\end{array}$ & $\begin{array}{l}-0,065 \\
(0,048)\end{array}$ & $\begin{array}{l}-0,248 * * * \\
(0,048)\end{array}$ & $\begin{array}{c}0,058 \\
(0,036)\end{array}$ \\
\hline América Latina & $\begin{array}{l}-5,408 * * * \\
(2,004)\end{array}$ & $\begin{array}{l}-0,338 \\
(1,589)\end{array}$ & $\begin{array}{l}-0,098 \\
(0,081)\end{array}$ & $\begin{array}{c}0,040 \\
(0,073)\end{array}$ & $\begin{array}{l}-0,120^{*} \\
(0,070)\end{array}$ & $\begin{array}{c}0,002 \\
(0,052)\end{array}$ & $\begin{array}{l}-0,107^{*} \\
(0,048)\end{array}$ & $\begin{array}{l}0,170 * * * \\
(0,043)\end{array}$ \\
\hline Parque vehicular & $\begin{array}{c}3,205^{*} \\
(1,836)\end{array}$ & $\begin{array}{l}-1,161 \\
(1,622)\end{array}$ & $\begin{array}{l}-0,022 \\
(0,089)\end{array}$ & $\begin{array}{c}0,008 \\
(0,060)\end{array}$ & $\begin{array}{l}-0,022 \\
(0,061)\end{array}$ & $\begin{array}{c}0,031 \\
(0,053)\end{array}$ & $\begin{array}{l}-0,090 * * \\
(0,042)\end{array}$ & $\begin{array}{l}-0,095 * * * \\
(0,025)\end{array}$ \\
\hline Sustituto & $\begin{array}{c}0,108 \\
(2,556)\end{array}$ & $\begin{array}{l}-1,495 \\
(1,997)\end{array}$ & $\begin{array}{l}-0,042 \\
(0,086)\end{array}$ & $\begin{array}{l}-0,061 \\
(0,121)\end{array}$ & $\begin{array}{l}-0,040 \\
(0,088)\end{array}$ & $\begin{array}{l}-0,045 \\
(0,061)\end{array}$ & $\begin{array}{l}-0,111 \\
(0,071)\end{array}$ & $\begin{array}{l}-0,365^{* * * *} \\
(0,046)\end{array}$ \\
\hline Sección cruzada & $\begin{array}{c}-11,588 * * * \\
(2,853)\end{array}$ & $\begin{array}{l}-3,091 \\
(4,533)\end{array}$ & $\begin{array}{l}-0,362^{* * * *} \\
(0,134)\end{array}$ & $\begin{array}{l}-0,148 * \\
(0,082)\end{array}$ & $\begin{array}{l}-0,356^{* * * *} \\
(0,089)\end{array}$ & $\begin{array}{l}-0,097 \\
(0,130)\end{array}$ & $\begin{array}{l}-0,526 * * * \\
(0,054)\end{array}$ & \\
\hline Nacional & $\begin{array}{l}-4,179 * * * \\
(1,566)\end{array}$ & $\begin{array}{c}0,082 \\
(1,424)\end{array}$ & $\begin{array}{l}-0,087 \\
(0,077)\end{array}$ & $\begin{array}{c}0,101 \\
(0,066)\end{array}$ & $\begin{array}{l}-0,084 * \\
(0,053)\end{array}$ & $\begin{array}{l}-0,095 * * \\
(0,045)\end{array}$ & $\begin{array}{l}-0,120 * * * \\
(0,042)\end{array}$ & $\begin{array}{l}-0,032 \\
(0,031)\end{array}$ \\
\hline Dinámico & $\begin{array}{l}-7,147 * * * \\
(1,543)\end{array}$ & $\begin{array}{l}-0,081 \\
(1,860)\end{array}$ & $\begin{array}{l}-0,198 * * * \\
(0,065)\end{array}$ & $\begin{array}{l}-0,039 \\
(0,059)\end{array}$ & $\begin{array}{l}-0,230 * * * \\
(0,051)\end{array}$ & $\begin{array}{l}-0,025 \\
(0,062)\end{array}$ & $\begin{array}{l}-0,365^{* * * *} \\
(0,049)\end{array}$ & $\begin{array}{c}0,032 \\
(0,037)\end{array}$ \\
\hline Constante & $\begin{array}{l}10,294 * * * \\
(1,446)\end{array}$ & $\begin{array}{c}0,776 \\
(1,827)\end{array}$ & $\begin{array}{l}0,951 * * * \\
(0,055)\end{array}$ & $\begin{array}{l}0,3178 * * * \\
(0,067)\end{array}$ & $\begin{array}{l}0,961 * * * \\
(0,044)\end{array}$ & $\begin{array}{l}0,297 * * * \\
(0,056)\end{array}$ & $\begin{array}{l}0,983 * * * \\
(0,028)\end{array}$ & $\begin{array}{l}0,356 * * * \\
(0,037)\end{array}$ \\
\hline $\begin{array}{l}\mathrm{N}^{\mathrm{o}} \text { de observaciones } \\
R^{2} \text { ajustado } \\
\text { estadístico } F\end{array}$ & 119 & 108 & $\begin{array}{l}119 \\
0,42 \\
9,55 * * *\end{array}$ & $\begin{array}{r}108 \\
0,09\end{array}$ & $\begin{array}{l}119 \\
54,38 \\
16,07 * * *\end{array}$ & $\begin{array}{r}108 \\
4,23 \\
1,55\end{array}$ & 106 & 101 \\
\hline $\begin{array}{l}\chi^{2} \\
\text { Root MSE } \\
\tau^{2} \\
I^{2}\end{array}$ & $1077,4^{* * *}$ & $2320,1 * * *$ & 0,26 & 0,19 & $\begin{array}{c}0,050 \\
95,20 \%\end{array}$ & $\begin{array}{c}0,022 \\
89,40 \%\end{array}$ & $484,4 * * *$ & $267,2 * * *$ \\
\hline Log-likelihood & $-411,84$ & $-339,96$ & & & & & & \\
\hline
\end{tabular}

Fuente: Elaboración propia.

Nota: Los valores entre paréntesis son los errores estándar. Los asteriscos ***, ** y * indican significancia al $1 \%, 5 \%$ y $10 \%$, respectivamente. $R^{2}$ ajustado es el coeficiente de determinación ajustado de la meta-regresión y mide la proporción de la varianza entre estudios explicada por las variables incluidas en el modelo. El estadístico $F$ prueba la hipótesis nula de que las variables incluidas en el modelo en conjunto son igual a cero $\left(\beta_{1}=\beta_{2}=\ldots=\beta_{k}=0\right)$, para el caso de los modelos de efectos aleatorios y de Hubber-White; mientras que para los modelos de efectos fijos y mixtos, la prueba de significancia conjunta se hace mediante la $\chi^{2}$. $\tau^{2}$ estima la varianza entre estudios para el caso del modelo de efectos aleatorios. $I^{2}$ indica la proporción de la variación observada en la magnitud de los efectos atribuible a la heterogeneidad entre los estudios. Root MSE es la raíz cuadrada del error cuadrático medio. SE corresponde al error estándar.

ocDE: Organización para la Cooperación y el Desarrollo Económicos.

CUADRO 10

Meta-regresión: determinantes de la elasticidad precio de la demanda de gasolinas

\begin{tabular}{|c|c|c|c|c|c|c|c|c|}
\hline \multirow{2}{*}{ Parámetro } & \multicolumn{2}{|c|}{ Efectos mixtos } & \multicolumn{2}{|c|}{ Hubber-White } & \multicolumn{2}{|c|}{ Efectos aleatorios } & \multicolumn{2}{|c|}{ Efectos fijos } \\
\hline & Largo plazo & Corto plazo & Largo plazo & Corto plazo & Largo plazo & Corto plazo & Largo plazo & Corto plazo \\
\hline $1 / S E$ & $\begin{array}{l}-0,324 * * * \\
(0,021)\end{array}$ & $\begin{array}{l}-0,087 * * * \\
(0,022)\end{array}$ & & & & & & \\
\hline OCDE & $\begin{array}{l}-1,382 * * * \\
(0,443)\end{array}$ & $\begin{array}{l}-2,548 \\
(2,359)\end{array}$ & $\begin{array}{l}-0,132 \\
(0,092)\end{array}$ & $\begin{array}{l}-0,031 \\
(0,039)\end{array}$ & $\begin{array}{l}-0,102 * * \\
(0,045)\end{array}$ & $\begin{array}{l}-0,071 * * \\
(0,034)\end{array}$ & $\begin{array}{l}-0,145^{* * * *} \\
(0,035)\end{array}$ & $\begin{array}{l}-0,064 * * * \\
(0,018)\end{array}$ \\
\hline América Latina & $\begin{array}{l}-1,426^{* *} \\
(0,575)\end{array}$ & $\begin{array}{l}-3,109 \\
(2,628)\end{array}$ & $\begin{array}{l}-0,151 * \\
(0,089)\end{array}$ & $\begin{array}{l}-0,022 \\
(0,058)\end{array}$ & $\begin{array}{l}-0,159^{* *} \\
(0,064)\end{array}$ & $\begin{array}{l}-0,059 \\
(0,039)\end{array}$ & $\begin{array}{c}0,035 \\
(0,041)\end{array}$ & $\begin{array}{l}-0,008 \\
(0,024)\end{array}$ \\
\hline Parque vehicular & $\begin{array}{c}0,723 * \\
(0,452)\end{array}$ & $\begin{array}{c}2,206 \\
(2,603)\end{array}$ & $\begin{array}{c}0,089 \\
(0,064)\end{array}$ & $\begin{array}{c}0,022 \\
(0,058)\end{array}$ & $\begin{array}{l}-0,062 \\
(0,045)\end{array}$ & $\begin{array}{c}0,037 \\
(0,038)\end{array}$ & $\begin{array}{c}0,006 \\
(0,035)\end{array}$ & $\begin{array}{c}0,016 \\
(0,017)\end{array}$ \\
\hline Sustituto & $\begin{array}{l}-0,380 \\
(0,397)\end{array}$ & $\begin{array}{l}-3,852 \\
(3,188)\end{array}$ & $\begin{array}{l}-0,123^{* *} \\
(0,054)\end{array}$ & $\begin{array}{l}-0,124 \\
(0,079)\end{array}$ & $\begin{array}{l}-0,112 * * \\
(0,046)\end{array}$ & $\begin{array}{l}-0,109 * * \\
(0,047)\end{array}$ & $\begin{array}{l}-0,131 * * * \\
(0,028)\end{array}$ & $\begin{array}{l}-0,251 * * * \\
(0,072)\end{array}$ \\
\hline Sección cruzada & $\begin{array}{l}-1,051 \\
(0,749)\end{array}$ & $\begin{array}{l}-2,232 \\
(5,736\end{array}$ & $\begin{array}{l}-0,320 * * * \\
(0,095)\end{array}$ & $\begin{array}{l}-0,319 * * * \\
(0,073)\end{array}$ & $\begin{array}{l}-0,252^{* * * *} \\
(0,088)\end{array}$ & $\begin{array}{l}-0,289 * * * \\
(0,088)\end{array}$ & $\begin{array}{l}-0,138 * * \\
(0,065)\end{array}$ & $\begin{array}{l}-0,372 * * * \\
(0,087)\end{array}$ \\
\hline Nacional & $\begin{array}{l}0,829 * * \\
(0,415)\end{array}$ & $\begin{array}{c}3,585 \\
(2,324)\end{array}$ & $\begin{array}{c}0,059 \\
(0,096)\end{array}$ & $\begin{array}{c}0,023 \\
(0,041)\end{array}$ & $\begin{array}{c}0,071 * \\
(0,042)\end{array}$ & $\begin{array}{c}0,033 \\
(0,034)\end{array}$ & $\begin{array}{l}0,167 * * * \\
(0,031)\end{array}$ & $\begin{array}{l}-0,047 * * \\
(0,019)\end{array}$ \\
\hline
\end{tabular}


Cuadro 10 (conclusión)

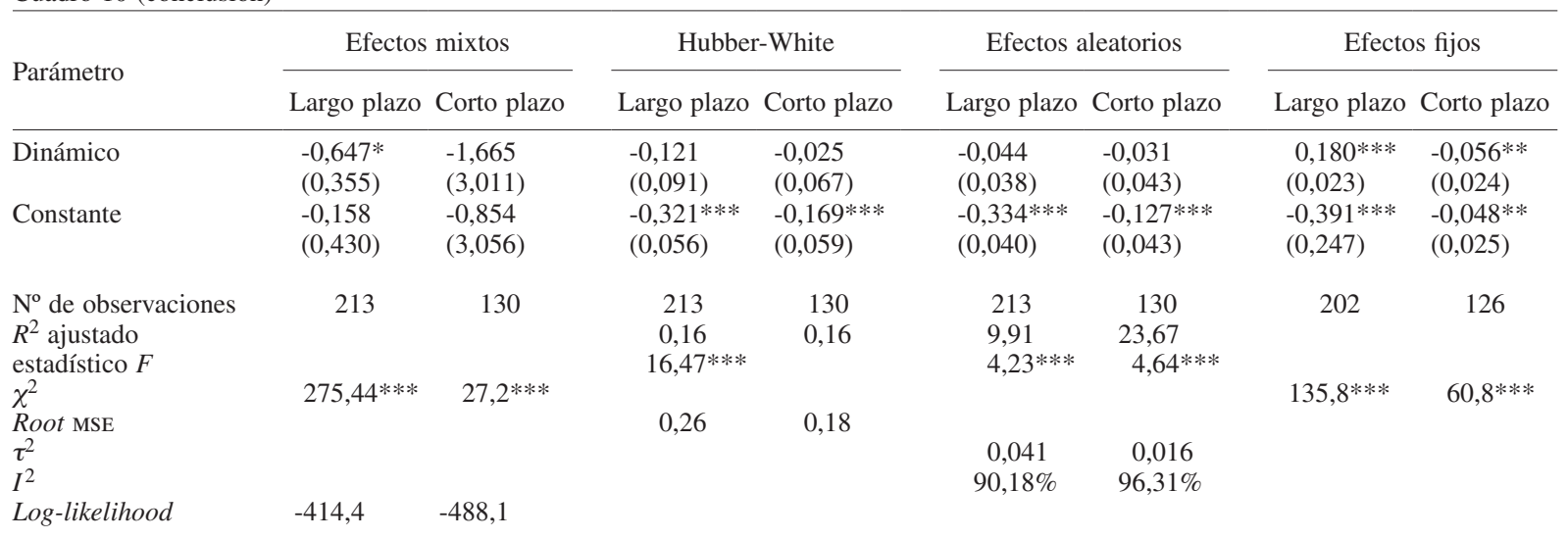

Fuente: Elaboración propia.

Nota: Los valores entre paréntesis son los errores estándar. ***, ** y * indican significancia al $1 \%, 5 \%$ y $10 \%$, respectivamente. $R^{2}$ ajustado es el coeficiente de determinación ajustado de la mea-regresión y mide la proporción de la varianza entre estudios explicada por las variables incluidas en el modelo. El estadístico $F$ prueba la hipótesis nula que las variables incluidas en el modelo en conjunto son igual a cero $\left(\beta_{1}=\beta_{2}=\ldots=\beta_{k}=0\right)$, para el caso de los modelos de efectos aleatorios y de Hubber-White; mientras que para los modelos de efectos fijos y mixtos, la prueba de significancia conjunta se hace a través de la $\chi^{2} \cdot \tau^{2}$ estima la varianza entre estudios para el caso del modelo de efectos aleatorios. $I^{2}$ indica la proporción de la variación observada en la magnitud de los efectos atribuible a la heterogeneidad entre los estudios. Root MSE es la raíz cuadrada del error cuadrático medio. SE corresponde al error estándar. OCDE: Organización para la Cooperación y el Desarrollo Económicos.

CUADRO 11

Resumen de las estimaciones de elasticidades ingreso y precio de la demanda de gasolinas

\begin{tabular}{|c|c|c|c|c|}
\hline \multirow{2}{*}{ Modelo } & \multicolumn{2}{|c|}{ Elasticidad ingreso } & \multicolumn{2}{|c|}{ Elasticidad precio } \\
\hline & Largo plazo & Corto plazo & Largo plazo & Corto plazo \\
\hline \multirow[t]{2}{*}{ Efectos fijos } & $0,551 * * *$ & $0,268 * * *$ & $-0,131 * * *$ & $-0,108 * * *$ \\
\hline & $(0,002)$ & $(0,001)$ & $(0,003)$ & $(0,002)$ \\
\hline \multirow[t]{2}{*}{ Efectos aleatorios } & $0,625 * * *$ & $0,303 * * *$ & $-0,397 * * *$ & $-0,204 * * *$ \\
\hline & $(0,031)$ & $(0,017)$ & $(0,015)$ & $(0,021)$ \\
\hline \multirow[t]{2}{*}{ Efectos mixtos } & $0,461 * * *$ & $0,267 * * *$ & $-0,314 * * *$ & $-0,104 * * *$ \\
\hline & $(0,017)$ & $(0,006)$ & $(0,014)$ & $(0,018)$ \\
\hline
\end{tabular}

Fuente: Elaboración propia.

Nota: Los valores entre paréntesis son los errores estándar. Los asteriscos ***, ** y * indican significancia al 1\%, 5\% y $10 \%$, respectivamente.

CUADRO 12

Elasticidad ingreso y precio de la demanda de gasolinas por región

\begin{tabular}{lcc}
\hline & Países de la ocDE & Resto del mundo \\
\hline Elasticidad ingreso & & 0,79 \\
$\quad$ Elasticidad de largo plazo & 0,55 & 0,29 \\
$\quad$ Elasticidad de corto plazo & 0,24 & 0,69 \\
Elasticidad precio & $-0,41$ & $-0,37$ \\
$\quad$ Elasticidad de largo plazo & $-0,22$ & $-0,20$ \\
Elasticidad de corto plazo & $-0,31$ \\
\hline
\end{tabular}

Fuente: Elaboración propia.

Nota: La estimación de la elasticidad ponderada por la desviación estándar fue realizada por medio del modelo de efectos aleatorios. En todos los casos, la prueba $Q$ rechaza la hipótesis nula de homogeneidad de las estimaciones. De igual manera, el estadístico $I^{2}$ indica, para las elasticidades ingreso y precio de largo y de corto plazo, que la proporción de la variación observada en la magnitud de los efectos atribuible a la heterogeneidad entre los estudios es mayor de 85\%. OCDE hace referencia a los países miembros de la Organización para la Cooperación y el Desarrollo Económicos, excluidos Chile y México. 
- Las magnitudes de las elasticidades ingreso y precio se modifican de acuerdo con las características y evolución del parque vehicular. De esta manera, la elasticidad ingreso y precio de largo plazo es menor en los estudios que incluyen el parque de vehículos que en aquellos donde no se incluye esta variable explicativa (véase el cuadro 13). En el corto plazo, se observa que la capacidad de respuesta es menor ante cambios en el ingreso y el precio. La reducción de la elasticidad ingreso de la demanda de gasolinas -al incluirse como variable explicativa la flota vehicular - es, parcialmente, consecuencia de la fuerte colinealidad entre la trayectoria del ingreso y de la flota vehicular. Asimismo, los cambios en las elasticidades precio sugieren la presencia de complejos procesos de ajuste de los consumidores.

- Las magnitudes de las elasticidades precio de la demanda de gasolinas se modifican al incluirse los precios de otros combustibles sustitutos en la especificación de la demanda de combustibles, como el etanol o biodiésel (véase el cuadro 14). Como es de esperar, la existencia de combustibles sustitutos hace que los consumidores tengan una mayor capacidad de respuesta, tanto a largo como a corto plazo, en la cantidad demandada ante cambios en el precio.

- Las magnitudes de las elasticidades ingreso y precio también son diferentes dependiendo de los métodos de estimación aplicados (Graham y Glaister, 2002; Espey, 1998). De esta manera, al realizar el meta-análisis considerando únicamente trabajos en los que se aplica el método de máxima verosimilitud (MV), se obtienen mayores elasticidades ingreso que al incluirse solo trabajos que utilizan mínimos cuadrados ordinarios (MCO) o el método generalizado de momentos (MGM) como métodos de estimación (véase el cuadro 15). Las elasticidades precio también son diferentes según el método de estimación empleado, lo que es consistente con los trabajos de Espey (1998) y Havranek y otros (2012).

\section{Elasticidad ingreso y precio de la demanda de gasolinas considerando el parque vehicular}

\begin{tabular}{lcc}
\hline & $\begin{array}{c}\text { Elasticidad considerando } \\
\text { el parque vehicular }\end{array}$ & $\begin{array}{c}\text { Elasticidad sin considerar } \\
\text { el parque vehicular }\end{array}$ \\
\hline Elasticidad ingreso & & 0,67 \\
$\quad$ Elasticidad de largo plazo & 0,49 & 0,30 \\
$\quad$ Elasticidad de corto plazo & 0,29 & $-0,40$ \\
Elasticidad precio & & $-0,21$ \\
$\quad$ Elasticidad de largo plazo & $-0,35$ & $-0,16$ \\
$\quad$ Elasticidad de corto plazo & $-0,25$ \\
\hline
\end{tabular}

Fuente: Elaboración propia.

Nota: La estimación de la elasticidad ponderada por la desviación estándar fue realizada por medio del modelo de efectos aleatorios. En todos los casos, la prueba $Q$ rechaza la hipótesis nula de homogeneidad de las estimaciones. El estadístico $I^{2}$ indica, para las elasticidades ingreso y precio de largo y de corto plazo, que la proporción de la variación observada en la magnitud de los efectos atribuible a la heterogeneidad entre los estudios es mayor de $85 \%$.

CUADRO 14

Elasticidad ingreso y precio de la demanda de gasolinas considerando precios de sustitutos

\begin{tabular}{lcc}
\hline & $\begin{array}{c}\text { Elasticidad considerando } \\
\text { el precio de sustitutos }\end{array}$ & $\begin{array}{c}\text { Elasticidad sin considerar } \\
\text { el precio de sustitutos }\end{array}$ \\
\hline Elasticidad ingreso & 0,58 & 0,62 \\
$\quad$ Elasticidad de largo plazo & 0,23 & 0,31 \\
$\quad$ Elasticidad de corto plazo & $-0,48$ & $-0,38$ \\
Elasticidad precio & $-0,32$ & $-0,16$ \\
$\quad$ Elasticidad de largo plazo & & \\
Elasticidad de corto plazo & & \\
\hline
\end{tabular}

Fuente: Elaboración propia.

Nota: La estimación de la elasticidad ponderada por la desviación estándar fue realizada por medio del modelo de efectos aleatorios. En todos los casos, la prueba $Q$ rechaza la hipótesis nula de homogeneidad de las estimaciones. El estadístico $I^{2}$ indica, para las elasticidades ingreso y precio de largo y de corto plazo, que la proporción de la variación observada en la magnitud de los efectos atribuible a la heterogeneidad entre los estudios es mayor de $85 \%$. 
Elasticidad ingreso y precio de la demanda de gasolinas por método de estimación

\begin{tabular}{lcc}
\hline & $\begin{array}{c}\text { Mínimos cuadrados } \\
\text { ordinarios (MCO) }\end{array}$ & $\begin{array}{c}\text { Máxima } \\
\text { verosimilitud }\end{array}$ \\
\hline Elasticidad ingreso & 0,62 & 0,67 \\
Elasticidad de largo plazo & 0,33 & 0,49 \\
Elasticidad de corto plazo & & 0,27 \\
Elasticidad precio & $-0,46$ & $-0,20$ \\
$\quad$ Elasticidad de largo plazo & $-0,20$ & $-0,08$ \\
Elasticidad de corto plazo & & $-0,11$ \\
\hline
\end{tabular}

Fuente: Elaboración propia.

Nota: La estimación de la elasticidad ponderada por la desviación estándar fue realizada por medio del modelo de efectos aleatorios. En todos los casos, la prueba $Q$ rechaza la hipótesis nula de homogeneidad de las estimaciones. El estadístico $I^{2}$ indica, para las elasticidades ingreso y precio de largo y de corto plazo, que la proporción de la variación observada en la magnitud de los efectos atribuible a la heterogeneidad entre los estudios es mayor de $85 \%$.

Los resultados del meta-análisis permiten inferir algunas conclusiones de política pública. En efecto, el consumo de gasolinas se vincula con diversas externalidades negativas, como los costos derivados del tráfico vehicular, la contaminación atmosférica y el cambio climático (Cnossen, 2005; Kayser, 2000). Existen, además, efectos colaterales con incidencia muy heterogénea, tales como cambios en los valores de las propiedades relacionados con la contaminación atmosférica y el ruido (Verhoef, 1994; Schipper, 1996). La magnitud de estos costos en América Latina es ciertamente significativa y probablemente seguirá aumentando de mantenerse el actual estilo de desarrollo (Parry y Strand, 2010; Hernández y Antón, 2013). Estos efectos negativos se concentran en las zonas urbanas, lo que resulta preocupante dado que en la actualidad aproximadamente el $80 \%$ de la población de América Latina se ubica en estas áreas y se espera que la población urbana en la región llegue a 640,1 millones de habitantes en el año $2050^{12}$.

Así, en América Latina —-donde se observa un acelerado crecimiento urbano y de las tasas de motorización- es frecuente que se excedan los estándares establecidos para la concentración de contaminantes atmosféricos. Esta situación se considera de gran riesgo para la salud pública, ya que existe evidencia de que un aumento de $10 \mu \mathrm{m} / \mathrm{m}^{3}$ (microgramos por metro cúbico) de material particulado (PM10) está asociado con un incremento de la morbilidad y mortalidad respiratoria (Cropper y otros, 1997; Lozano, 2004).

12 De acuerdo con las proyecciones de población del Centro Latinoamericano y Caribeño de Demografía (CELADE)-División de Población de la CEPAL.
De este modo, la elasticidad ingreso de la demanda media de gasolinas derivada del meta-análisis sugiere que un crecimiento económico continuo, bajo las actuales circunstancias, se traducirá en un aumento sustancial del consumo de gasolinas con sus consecuentes efectos colaterales negativos, principalmente en zonas urbanas. Incluso más, la evidencia disponible del meta-análisis muestra que ambas elasticidades ingreso son mayores en América Latina que en países desarrollados, usando como muestra a los países de la oCDE (excluidos Chile y México). Ello refleja que el estilo de crecimiento económico o su fase actual en la región están estrechamente ligados al consumo de gasolinas, mientras que en los países desarrollados se ha conseguido desacoplar ligeramente esta relación. La evidencia del meta-análisis denota, además, que las elasticidades ingreso se modifican con la inclusión de la flota vehicular. Parte de este cambio en la elasticidad ingreso de la demanda de gasolinas se puede explicar por la estrecha vinculación que existe entre el ingreso y la demanda de vehículos, que induce cierta multicolinealidad en la estimación. Así, el actual estilo de desarrollo en América Latina está consolidando una demanda creciente de un transporte privado que se refleja en el rápido crecimiento del parque vehicular. Todo ello sugiere que, bajo las actuales circunstancias, es muy probable que se intensifiquen las externalidades negativas ocasionadas por el transporte y el consumo de gasolinas en las áreas urbanas en la región.

Las elasticidades de corto plazo son menores que aquellas de largo plazo, lo que sugiere la presencia de costos de ajuste y de información en los agentes económicos que retrasan su respuesta al aumento del precio de las gasolinas. Existe incluso evidencia internacional de que los consumidores tienen una respuesta asimétrica a los cambios en los precios (Gately, 1992). 
La elasticidad precio de la demanda de gasolinas derivada del meta-análisis para América Latina sugiere que un impuesto a su consumo tiene una repercusión negativa, pero que sin embargo este efecto es pequeño y difícilmente será suficiente para controlar el aumento del consumo en una trayectoria de rápido crecimiento económico. La baja elasticidad precio de la demanda de gasolinas se debe, parcialmente, a que existen menos sustitutos adecuados al transporte privado en la región que en los países de la OCDE. De este modo, para acrecentar la sensibilidad del consumo a los precios relativos deben fomentarse alternativas reales al transporte privado.

Existe actualmente una amplia gama de instrumentos económicos y regulaciones que se aplican para controlar o inducir la trayectoria de la demanda de gasolinas. Por ejemplo, hay impuestos tales como al registro de autos, al uso de la infraestructura vial, al conjunto de las externalidades que ocasiona el transporte (contaminación atmosférica, ruido, accidentes o congestionamiento), a las gasolinas, al uso de las vías, a la importación de autos dependiendo de su cilindraje o características, además de sistemas de permisos comercializables o costos económicos adicionales que se reflejan en el valor de los seguros o tarifas por estacionamiento ${ }^{13}$ (Brons y otros, 2008; Markandya y Shibli, 1995). Existen, además, importantes procesos destinados a hacer más rigurosas las regulaciones sobre el transporte, que incluyen normas de eficiencia (kilómetros por litro de gasolina), ruido y normas de circulación por días o zonas (Comisión Europea, 2014; Alcaldía Mayor de Bogotá, 2000).

El uso de políticas fiscales respecto del consumo de gasolinas es, en todo caso, un instrumento relevante para controlar e inducir un comportamiento específico. Por ejemplo, la recaudación de impuestos relacionada con el medioambiente en los países de la OCDE representa alrededor de dos puntos porcentuales del PIB, aunque con un comportamiento heterogéneo relevante entre países. Por el contrario, en América Latina, en especial en países productores de hidrocarburos, persiste una tendencia a subsidiar productos derivados del petróleo.

El conjunto de la evidencia presentada sugiere que una estrategia que pretenda controlar el consumo de gasolinas debe incluir al menos los siguientes ejes:

\footnotetext{
13 Incluso se han utilizado sistemas para restringir el uso del auto en ciertas zonas sobre la base de instrumentos económicos, como en la ciudad de Boston (Gómez-Ibáñez y Futh, 1980).
}

i) Un aumento en el precio de las gasolinas, atendiendo a que el precio actual está en muchos casos subsidiado o no se incorporan en él las externalidades que ocasiona su consumo (Cnossen, 2005; Hernández y Antón, 2013).

ii) La aplicación de un conjunto de regulaciones que incidan en el consumo de gasolinas ${ }^{14}$ y sean consistentes con la estrategia de precios y las regulaciones. En estas regulaciones se pueden incluir medidas como licencias especiales, zonificación de tráfico, precios por congestión, restricciones de zonas de la ciudad para vehículos, mejoras del transporte público y medidas que se apliquen solo a los vehículos privados que tienen efectos en la congestión (Van den Bergh y otros, 2010).

iii) Estrategias explícitas para elevar la elasticidad precio de la demanda de gasolinas. Ello implica el desarrollo de una infraestructura vial moderna, eficiente y segura que privilegie el transporte público. El desarrollo de esta infraestructura puede además tener efectos positivos en el ingreso regional (Rietveld, 1994; Verhoef, 1994).

iv) Desarrollo de estrategias específicas de movilidad, que incluyen construcción de ciclovías, áreas peatonales y otras formas de transporte, además del desarrollo de una estrategia de densificación y uso del suelo urbano que permita optimizar el empleo del transporte masivo y reducir las distancias de recorrido. Por ejemplo, en un meta-análisis Phillips y Goss (1995) muestran controlando por servicios públicos, que el aumento de impuestos en determinada área metropolitana disminuye la actividad económica. Ello significa que es posible incidir en la conformación urbana y, por consiguiente, en las necesidades de movilidad a través del uso de diversos instrumentos económicos.

v) Considerar que las políticas públicas no inducen ajustes automáticos y que, en consecuencia, existe un rezago temporal antes de obtener sus efectos totales, como lo muestran las diferencias entre las elasticidades ingreso y precio de corto y de largo plazo.

\footnotetext{
${ }^{14}$ Por ejemplo, Goldberg (1998) muestra que el sistema de regulación de estándares de eficiencia en los Estados Unidos de América, conocido como normas corporativas de economía de combustible en los automóviles (CAFE, por sus siglas en inglés), puede ser complementario a una estrategia de precios.
} 


\section{IV}

\section{Conclusiones y comentarios generales}

La evidencia internacional sintetizada en este estudio permite constatar que existe una alta diversidad y volatilidad en las estimaciones de las elasticidades ingreso y precio de la demanda de gasolinas, así como la presencia de un comportamiento asimétrico en su distribución que sugiere un sesgo de publicación. Las estimaciones y las pruebas econométricas realizadas (FAT-PET-MRA) muestran que se rechaza la hipótesis nula de que las diferencias en las estimaciones son solo consecuencia de error de muestreo, que existe una heterogeneidad genuina y que, por lo tanto, es necesario utilizar un modelo de efectos mixtos en la meta-regresión.

Las estimaciones de la meta-regresión indican que las elasticidades ingreso y precio de la demanda de gasolinas son estadísticamente significativas, esto es, la media de las elasticidades de corto y largo plazo es diferente de cero incluso después de corregir por el sesgo de publicación y que, además, existe un sesgo positivo en la elasticidad ingreso de largo plazo y un sesgo negativo en las elasticidades precio de corto y largo plazo. Así, las estimaciones de la meta-regresión sugieren, con resultados para toda la muestra de estudios, elasticidades ingreso medias de 0,26 para el corto plazo y de 0,46 para el largo plazo. Asimismo, estas estimaciones sugieren elasticidades precio de corto y largo plazo de $-0,10$ y $-0,31$, respectivamente.

La volatilidad en las estimaciones es, desde luego, consecuencia de una multiplicidad de factores. La metaregresión señala que las estimaciones de las elasticidades ingreso se modifican atendiendo al nivel de desarrollo de los países, el parque vehicular, el tipo de datos utilizados, la zona de estimación y la estructura dinámica de los modelos, entre otros factores. Por su parte, la evidencia sobre las elasticidades precio muestra que existe también una volatilidad asimétrica que se origina tanto en sesgos de publicación, como en otros factores sistemáticos. La meta-regresión denota que las elasticidades precio de la demanda de gasolinas se modifican con la región o el parque de vehículos, entre otros factores.

Ello permite destacar que la media de la elasticidad ingreso de la demanda de gasolinas en América Latina es mayor que la de los países de la OCDE, mientras que la elasticidad precio media es más inelástica en la región que en los países de la OCDE. Esto es consecuencia de una compleja matriz de factores e interacciones. No obstante, puede argumentarse que la alta elasticidad ingreso y la baja elasticidad precio son reflejo de un mismo fenómeno general: el estilo y la fase del desarrollo económico de América Latina, con precios relativos y la conformación de un conjunto de opciones y modalidades de transporte que no representan un sustituto razonable al transporte privado. En efecto, en la región se observa - ajustando por precios- que el crecimiento económico está conformando nuevos grupos de consumidores que abandonan paulatinamente el transporte público por el transporte privado, en parte, porque el transporte público no satisface sus requerimientos de movilidad en términos de seguridad, comodidad y tiempos de recorrido.

Las consecuencias de este incremento del consumo de gasolinas se traducen en la conformación, especialmente en las zonas urbanas, de agudas externalidades negativas, tales como contaminación atmosférica, ruido, accidentes viales, congestionamiento y el cambio climático. Ello hace aconsejable aplicar un impuesto que permita internalizar los costos que ocasiona el consumo de gasolinas. Sin embargo, las elasticidades precio inferidas para América Latina sugieren que la respuesta al alza del precio es poco sensible, de modo que - en un entorno de rápido crecimiento- es muy probable que el consumo continúe aumentado. En todo caso, las bajas elasticidades precio pueden utilizarse por motivos recaudatorios. De este modo, una estrategia que procure contribuir al control de la demanda de gasolinas requiere, además, una estrategia de precios relativos o impuestos que reflejen el costo real de su consumo, medidas regulatorias tales como estándares de emisiones por kilómetro recorrido, limitaciones al tráfico vehicular, una infraestructura de transporte público adecuada, acompañada de ciclovías y áreas confinadas para peatones. Además, en un ámbito más general, se requiere la conformación de un estilo de desarrollo urbano consistente con estas medidas. Así, la creación de sistemas eficientes de transporte público masivo, mejoras en la calidad y el rendimiento de los combustibles, y progresos tecnológicos en la industria automotriz pueden no solo contribuir a mitigar las emisiones, sino también aportar beneficios en salud, menor accidentalidad y reducción de los tiempos de traslado.

El subsidio a las gasolinas es una política común en muchos de los países de América Latina; sin embargo, este constituye un incentivo perverso para los objetivos 
medioambientales, ya que estimula el consumo de un bien que induce una externalidad negativa. En este contexto, se requiere no solo desmontar los subsidios a los combustibles, sino que al momento de fijar su precio se tengan en cuenta los costos sociales de su consumo. La anulación de estos subsidios ocasionará no solo un aumento en el precio de las gasolinas, sino también de los demás bienes. Por ello, sería necesario que esta política se acompañe de programas que flexibilicen la carga en los sectores de menores ingresos, que pueden verse afectados por un alza del precio de las gasolinas debido a incrementos en el precio de los alimentos o del transporte público, requiriendo ser compensados adecuadamente.

\section{Bibliografía}

Abreu, M. y R. Florax (2005), "A meta-analysis of B-convergence: the legendary 2\%", Journal of Economic Surveys, vol. 19, $\mathrm{N}^{\circ}$ 3, Wiley.

Alcaldía Mayor de Bogotá (2000), Decreto 1099 de 2000. Por el cual se toman medidas para el mejor ordenamiento del tránsito de vehículos en las vías públicas de Bogotá, D.C., Bogotá.

Alves, D. y R. Bueno (2003), "Short-run, long-run and cross elasticities of gasoline demand in Brazil", Energy Economics, vol. 25, $\mathrm{N}^{\circ} 2$, Amsterdam, Elsevier.

Angrist, J.D. y J.S. Pischke (2009), Mostly Harmless Econometrics: An Empiricist's Companion, Princeton, Princeton University Press.

Apostol, T.M. (1967), Calculus, vol. 1, John Wiley \& Sons.

Bentzen, J. (1994), "An empirical analysis of gasoline demand in Denmark using cointegration techniques", Energy Economics, vol. 16, $\mathrm{N}^{\circ} 2$, Amsterdam, Elsevier.

Borenstein, M. y otros (2009), Introduction to Meta-Analysis, John Wiley and Sons.

Brons, M. y otros (2008), "A meta-analysis of the price elasticity of gasoline demand. A SUR approach", Energy Economics, vol. $30, \mathrm{~N}^{\circ} 5$, Amsterdam, Elsevier.

Calthrop, E. y S. Proost (1998), "Road transport externalities", Environmental and Resource Economics, vol. 11, $\mathrm{N}^{\circ} 3-4$, Springer.

Cnossen, S. (2005), Theory and Practice of Excise Taxation: Smoking, Drinking, Gambling, Polluting, and Driving, Nueva York, Oxford University Press.

Comisión Europea (2014), Comunicación de la Comisión al Parlamento Europeo con arreglo al artículo 294, apartado 6, del Tratado de Funcionamiento de la Unión Europea sobre la posición del Consejo relativa a la adopción de un reglamento del Parlamento Europeo y del Consejo sobre el nivel sonoro de los vehículos de motor (COM/2014/0107), Bruselas.

Cropper, M. y otros (1997), "The health benefits of air pollution control in Delhi", American Journal of Agricultural Economics, vol. 79, $\mathrm{N}^{\circ}$ 5, Agricultural and Applied Economics Association.

Cumming, G. (2012), Understanding the New Statistics: Effect Sizes, Confidence Intervals, and Meta-Analysis, Nueva York, Routledge

Dahl, C. (2012), "Measuring global gasoline and diesel price and income elasticities", Energy Policy, vol. 41, Amsterdam Elsevier.

(1992), "A survey of energy demand elasticities for the developing world", Journal of Energy Finance \& Development, vol. 18, $\mathrm{N}^{\circ} 1$, Amsterdam, Elsevier.

Deaton, A. y J. Muellbauer (1980), Economics and Consumer Behavior, Cambridge, Cambridge University Press.

Doucouliagos, H. y T.D. Stanley (2009), "Publication selection bias in minimum-wage research? A meta-regression analysis", British Journal of Industrial Relations, vol. 47, $\mathrm{N}^{\circ} 2$, Londres, London School of Economics and Political Science.

Egger, M. y otros (1997), "Bias in meta-analysis detected by a simple, graphical test", British Medical Journal, vol. 315, № 7109 , BMJ Publishing Group.
Espey, M. (1998), "Gasoline demand revisited: an international meta-analysis of elasticities", Energy Economics, vol. 20, Nㅜ 3, Amsterdam, Elsevier.

Galindo, L.M. (2008), Estudio sobre la instrumentación de medidas de eficiencia energética y uso de biocombustibles en el sector transporte y su impacto en la calidad del aire en México. Informe Final, Ciudad de México, Universidad Nacional Autónoma de México.

(2005), "Short- and long-run demand for energy in Mexico: a cointegration approach", Energy Policy, vol. 33, $\mathrm{N}^{\circ}$, Amsterdam, Elsevier.

Gately, D. (1992), "Imperfect price-reversibility of world oil demand", Energy Journal, vol. 14, N ${ }^{\circ}$ 4, International Association for Energy Economics.

Goldberg, P.K. (1998), "The effects of the corporate average fuel economy standards in the U.S.", Journal of Industrial Economics, vol. 46, $\mathrm{N}^{\circ} 1$, Wiley.

Gómez-Ibáñez, J.A. y G.R. Futh (1980), "Downtown auto restraint policies. The costs and benefits for Boston", Journal of Transport Economics and Policy, vol. 14, $\mathrm{N}^{\circ}$ 2, Universidad de Bath/London School of Economics and Political Science.

Goodwin, P.B. (1992), "A review of new demand elasticities with special reference to short and long run effects of price changes", Journal of Transport Economics and Policy, vol. 26, $\mathrm{N}^{\circ} 2$, Universidad de Bath/London School of Economics and Political Science.

Graham, D. y S. Glaister (2002), "The demand for automobile fuel a survey of elasticities", Journal of Transport Economics and Policy, vol. 36, $\mathrm{N}^{\circ}$ 1, Universidad de Bath/London School of Economics and Political Science.

Hanly, M. y otros (2002), Review of Income and Price Elasticities in the Demand for Road Traffic, Londres, University College London.

Havranek, T. y otros (2012), "Demand for gasoline is more priceinelastic than commonly thought", Energy Economics, vol. 34, $\mathrm{N}^{\circ}$ 1, Amsterdam, Elsevier.

Heckman, J.J. (1979), "Sample selection bias as a specification error", Econometrica, vol. 47, $\mathrm{N}^{\circ} 1$, Nueva York, The Econometric Society.

Hernández, F. y A. Antón (2013), "Optimal gasoline tax in developing, oil-producing countries: the case of Mexico", Documento de Trabajo, $\mathrm{N}^{\circ}$ 555, Ciudad de México, Centro de Investgación y Docencia Económicas (CIDE).

Kayser, H.A. (2000), "Gasoline demand and car choice: estimating gasoline demand using household information", Energy Economics, vol. 22, $\mathrm{N}^{\circ} 3$, Amsterdam, Elsevier.

Kim, Y.D. y otros (2011), "The empirical effects of a gasoline tax on $\mathrm{CO}_{2}$ emissions reductions from transportation sector in Korea", Energy Policy, vol. 39, $\mathrm{N}^{\circ} 2$, Amsterdam, Elsevier.

Lipsey, M.W. y D.B. Wilson (2001), Practical Meta-Analysis, Thousand Oaks, Sage Publications.

Lozano, N. (2004), "Air pollution in Bogotá, Colombia: a concentration-response approach", Desarrollo y Sociedad, $\mathrm{N}^{\circ}$ 54, Bogotá, Universidad de los Andes. 
Markandya, A. y A. Shibli (1995), "Industrial pollution control policies in Asia", Environment Discussion Paper Series, $\mathrm{N}^{\circ} 3$, Cambridge, Massachusetts, Harvard Institute for International Development.

Parry, I. y J. Strand (2010), "International fuel tax assessment: an application to Chile", Discussion Papers, $\mathrm{N}^{\circ} 10-07$, Washington, D.C., Resources for the Future.

Paterson, B.L. y C. Canam (2001), Meta-Study of Qualitative Health Research: A Practical Guide to Meta-Analysis and MetaSynthesis, SAGE Publications.

Phillips, J.M. y E.P. Goss (1995), "The effect of state and local taxes on economic development: a meta-analysis", Southern Economic Journal, vol. 62, $\mathrm{N}^{\circ}$ 2, Chattanooga, Southern Economic Association.

Pock, M. (2007), "Gasoline and diesel demand in Europe: new insights", Economics Series, $N^{\circ} 202$, Institute for Advanced Studies.

Reyes, M.O. (2009), "La demanda de gasolinas en México: efectos y alternativas ante el cambio climático", Documento de Trabajo, Barcelona, Universidad Autónoma de Barcelona.

Rietveld, P. (1994), "Spatial economic impacts of transport infrastructure supply", Transport Research, vol. 28, $\mathrm{N}^{\circ} 4$, Amsterdam, Elsevier

Sáez, M. y otros (2001), "Comparing meta-analysis and ecologicallongitudinal analysis in time-series studies. A case study of the effects of air pollution on mortality in three Spanish cities", Journal of Epidemiology \& Community Health, vol. 55, $\mathrm{N}^{\circ} 6$.

Schipper, Y.J.J. (1996), "On the valuation of aircraft noise: a metaanalysis", Zurich, European Regional Science Association [en línea] http://www-sre.wu.ac.at/ersa/ersaconfs/ersa96/ SESSION.D/d217.pdf.

Stanley, T.D. (2008), "Meta-regression methods for detecting and estimating empirical effects in the presence of publication selection”, Oxford Bulletin of Economics and Statistics, vol. 70, $\mathrm{N}^{\circ} 1$, Oxford, Universidad de Oxford.

(2005), "Beyond publication bias", Journal of Economic Surveys, vol. 19, Nㅜ 3, Wiley.
(2001), "Wheat from chaff: meta-analysis as quantitative literature review", Journal of Economic Perspectives, vol. 15, $\mathrm{N}^{\circ}$ 3, Nashville, Tennessee, American Economic Association.

Stanley, T.D. y H. Doucouliagos (2012), Meta-Regression Analysis in Economics and Business, Routledge.

(2011), "Meta-regression approximations to reduce publication selection bias", Economics Series, N ${ }^{\circ} 2011 / 4$, Deakin University.

Stanley, T.D. y S.B. Jarrell (1989), "Meta-regression analysis: a quantitative method of literature surveys", Journal of Economic Surveys, vol. 3, $\mathrm{N}^{\circ} 2$, Wiley.

Steenhof, P. y otros (2006), "Greenhouse gas emissions and the surface transport of freight in Canada", Transportation Research Part D: Transport and Environment, vol. 11, $\mathrm{N}^{\circ} 5$, Amsterdam, Elsevier.

Sterne, J.A.C. (2009), Meta-Analysis in STATA: An Updated Collection from the STATA Journal, Texas, STATA Press.

Sterne, J.A.C. y M. Egger (2001), "Funnel plots for detecting bias in meta-analysis: guidelines on choice of axis", Journal of Clinical Epidemiology, vol. 54, $\mathrm{N}^{\circ} 10$, Amsterdam, Elsevier.

Sterne, J.A.C. y otros (2000), "Publication and related bias in meta-analysis: power of statistical tests and prevalence in the literature", Journal of Clinical Epidemiology, vol. 53, $\mathrm{N}^{\circ} 11$, Amsterdam, Elsevier.

Sterner, T., C. Dahl y M. Franzén (1992), "Gasoline tax policy, carbon emissions and the global environment", Journal of Transport Economics and Policy, vol. 26, $\mathrm{N}^{\circ}$ 2, Universidad de Bath London School of Economics and Political Science.

Van den Bergh, J.C. y otros (2010), Meta-Analysis in Environmental Economics, Springer Science Business Media, B.V.

Varian, H.R. (1993), Análisis microeconómico, Barcelona, Antoni Bosch Editor.

Verhoef, E. (1994), "External effects and social costs of road transport", Transportation Research Part A: Policy and Practice, vo1. 28, $\mathrm{N}^{\circ}$ 4, Amsterdam, Elsevier.

Williams, R.L. (2000), "A note on robust variance estimation for cluster-correlated data", Biometrics, vol. 56, $\mathrm{N}^{\circ} 2$, Wiley.

Wooldridge, J.M. (2002), Econometric Analysis of Cross Section and Panel Data, Cambridge, Massachusetts, The MIT Press. 